\title{
Neural mechanisms underlying respiratory regulation within the preBötzinger complex of the rabbit
}

\author{
Elenia Cinelli, Donatella Mutolo, Tito Pantaleo, Fulvia Bongianni * \\ Dipartimento di Medicina Sperimentale e Clinica, Sezione Scienze Fisiologiche, Università Degli Studi di Firenze, Viale G.B. Morgagni 63, Firenze, 50134, Italy
}

\section{A R T I C L E I N F O}

Edited by M. Dutschmann

\section{Keywords:}

preBötzinger complex

Respiratory rhythm generation

Neuromodulators

5 -HT

Opioids

Substance P

\begin{abstract}
A B S T R A C T
The preBötzinger complex (preBötC) is a medullary area essential for normal breathing and widely recognized as necessary and sufficient to generate the inspiratory phase of respiration. It has been studied mainly in rodents. Here we report the main results of our studies revealing the characteristics of the rabbit preBötC identified by means of neuronal recordings, D,L-homocysteic acid microinjections and histological controls. A crucial role in the respiratory rhythmogenesis within this neural substrate is played by excitatory amino acids, but also GABA and glycine display important contributions. Increases in respiratory frequency are induced by microinjections of neurokinins, somatostatin as well by serotonin $(5-\mathrm{HT})$ through an action on $5-\mathrm{HT}_{1 \mathrm{~A}}$ and $5-\mathrm{HT}_{3}$ receptors or the disinhibition of a GABAergic circuit. Respiratory depression is observed in response to microinjections of the $\mu$-opioid receptor agonist DAMGO. Our results show similarities and differences with the rodent preBötC and emphasize the importance of comparative studies on the mechanisms underlying respiratory rhythmogenesis in different animal species.
\end{abstract}

\section{Introduction}

One of the most intriguing problems in neuroscience concerns the understanding of how the respiratory activity is generated and controlled. Early studies determined the location and role of respiratoryrelated central neural structures through lesioning, stimulation, anatomical mapping, and neuronal recordings (review by Von Euler, 1986, 1997). Subsequent studies using pharmacological and electrophysiological tools as well as genetics and molecular biology identified discrete neural substrates that operate within the ponto-medullary respiratory network (Smith et al., 2009, 2013; Pagliardini et al., 2011; Ramirez et al., 2012; Dhingra et al., 2019, 2020; Ashhad and Feldman, 2020; for review see Alheid et al., 2004; Dutschmann and Dick, 2012; Feldman et al., 2013; Jones and Dutschmann, 2016; Del Negro et al., 2018).

The motor pattern during normal breathing is generally considered to consist of three phases: inspiration, postinspiration or expiratory phase 1 and expiratory phase 2 (active expiration). Respiratory rhythmogenesis in adult mammals results from synaptic interactions between neurons located in the ventral respiratory column (VRC) in the medulla oblongata (Richter et al., 1986; Von Euler, 1986; Bianchi et al., 1995; Haji et al., 2000; McCrimmon et al., 2000; Alheid et al., 2002; Feldman and Del Negro, 2006; Alheid and McCrimmon, 2008; Doi and Ramirez, 2008; Del Negro et al., 2018). A subregion critical for inspiratory rhythm generation, the preBötzinger complex (preBötC), was initially identified in vitro in neonatal rats (Feldman et al., 1990; Smith et al., 1991) and has since been described in vivo in several animal species, including humans (e.g. Smith et al., 1991; Johnson et al., 1994, 2001; Schwarzacher et al., 1995, 2011; Ramirez et al., 1998). Other rhythmogenic circuits in the VRC may contribute to respiratory pattern formation: the retrotrapezoid nucleus/parafacial respiratory group (RTN/pFRG) was initially described as the main location of preinspiratory neurons (Onimaru and Homma, 1992, 2003), but has more recently been suggested as an expiratory oscillator contributing to active expiration (Janczewski and Feldman, 2006; Abdala et al., 2009; Abbott et al., 2011). Other components of the RTN/pFRG have also been characterized as responsible for central $\mathrm{CO}_{2}$ chemosensitivity (Mulkey et al., 2004; Guyenet, 2012; Guyenet et al., 2012; Wang et al., 2013; Del Negro et al., 2018). More recently, evidence has been provided in the mouse that a medullary region, named Postinspiratory Complex (PiCo), is characterized by rhythm-generating properties and is necessary and sufficient to generate postinspiratory activity (Anderson et al., 2016). A "triple oscillator model" has been proposed in which inspiration, postinspiration and active expiration are generated by three distinct oscillators, i.e. the

\footnotetext{
* Corresponding author.

E-mail address: fulvia.bongianni@unifi.it (F. Bongianni).
} 
preBötC, the PiCo and the pFRG (Anderson and Ramirez, 2017; Del Negro et al., 2018). In rats a corresponding area was identified in the intermediate reticular nucleus, however, inhibition of this area had no effect on inspiratory duration (Toor et al., 2019). Toor et al. suggested that these neurons were not involved in the inspiratory off-switch mechanism of the respiratory rhythm generator but rather serve as premotor neurons to motoneurons controlling airway patency and swallow. Similarly, local field potentials did not show highly synchronized activity in the intermediate reticular nucleus during the phase switch from inspiration to postinspiration (Dhingra et al., 2020), which supports the notion that the area does not contribute to respiratory rhythm generation.

Other regions with a respiratory function have been described. They include the dorsal respiratory group, largely corresponding to the ventrolateral nucleus of the solitary tract (NTS), and portions of the VRC such as the expiratory Bötzinger complex (BötC), the inspiratory portion of the VRG and the caudal expiratory component of the VRG. Respiration-related neurons are also present in the dorsolateral pons at the level of the parabrachial (PB) complex and Kölliker-Fuse (KF) nuclei. The main respiratory function of these nuclei is the regulation of the inspiratory-expiratory phase transition and the dynamic control of upper airway patency during the respiratory cycle (see e.g. Dutschmann and Dick, 2012; Dhingra et al., 2017; Ramirez and Baertsch, 2018). Recent studies in rodents have demonstrated that preBötC somatostatin (SST)-expressing glutamatergic neurons and neurons that express the glycine transporter GlyT2 extensively project to multiple brainstem regions involved in the control of breathing (Tan et al., 2010; Yang and Feldman, 2018). Interestingly, many brainstem regions, including the contralateral preBötC, the BötC, the PB/KF, the RTN/pFRG and the NTS, have reciprocal connections with excitatory and inhibitory preBötC neurons (Yang et al., 2020). In addition, suprapontine regions such as the superior colliculus, the dorsomedial and lateral hypothalamus, and the zona incerta also have similar reciprocal projections that may represent potential direct pathways for volitional, emotional and physiological control of breathing (Yang et al., 2020).

Despite substantial advances in the understanding of the anatomical and neurophysiological basis of respiratory rhythm and pattern generation, the underlying neural mechanisms have not yet been exhaustively defined. Since breathing is a vital function, it seems plausible that the respiratory central pattern generator (CPG) does not rely on a single neural substrate, but engages distributed neuronal populations within the different brainstem respiratory compartments and is characterized by a great deal of redundancy and degeneracy (see e.g. Von Euler, 1997; Mutolo et al., 2002; Smith et al., 2007; Jones and Dutschmann, 2016; Dhingra et al., 2019, 2020).

Our research activity has been devoted, to a large extent, to investigate the rostral VRC in the rabbit and in particular the preBötC region. Although the bulk of the basic knowledge of the mammalian respiratory network derives from experiments on cats and rodents, rabbits have also been widely used in studies of the control of breathing and of the localization of respiration-related regions (Gromysz and Karczewski, 1981; Yamamoto and Lagercrantz, 1985; Jiang and Shen, 1991; Stucke et al., 2015; for reviews see Von Euler, 1986; Bianchi et al., 1995; Hilaire and Duron, 1999). We used several criteria to identify the preBötC in the rabbit and its analogy with the preBötC described in other animal species. Extracellular recordings demonstrated predominantly expiratory neurons with prevailing augmenting discharge patterns in the BötC, inspiratory neurons with prevailing augmenting discharge patterns in the rostral VRG as well as a mix of different types of respiration-related neurons within a subregion located between the BötC and the rostral VRG, corresponding to the preBötC. This region contains expiratory neurons with augmenting discharge patterns, expiratory neurons with decrementing discharge patterns or postinspiratory neurons, inspiratory neurons with augmenting discharge patterns, and phase-spanning neurons. Some phase-spanning neurons started firing during the expiratory phase (expiratory-inspiratory) and reached a maximum rate during inspiration, others started firing during inspiration (inspiratory-expiratory) and reached a firing peak at the transition from inspiration to expiration (Fig. 1A; see e.g. Mutolo et al., 2002; Bongianni et al., 2008, 2010). These neuronal activities matched the neuronal discharge patterns described in the preBötC in other species (e.g. Connelly et al., 1992; Rekling and Feldman, 1998; Schwarzacher et al., 1995; St Jacques and St John, 1999; Sun et al., 1998).

The location of the preBötC was identified through tachypneic responses to microinjections of D,L-homocysteic acid (DLH; Fig. 1B). Postmortem histology confirmed the location in the rabbit, which is ventro-medial to the rostral portion of the nucleus ambiguus (Fig. 1C and Fig. 2D; see Mutolo et al., 2002, 2005; Bongianni et al., 2008, 2010; Iovino et al., 2019; Cinelli et al., 2020). Experiments were performed on $\alpha$-chloralose-urethane anaesthetized, vagotomized, paralyzed and artificially ventilated rabbits. Bilateral microinjections were performed into the VRG regions as defined by neuronal recordings. In each experiment, recordings of neuronal activity preceded drug microinjections. To restrict the spread of the injectate and thereby the number of neurons affected, relatively small volumes (30-50 nl) of drugs were bilaterally injected. Antagonist concentrations were always supramaximal. Agonist concentrations were selected in preliminary trials and were just above the minimum effective concentration capable to produce obvious and consistent effects. All drug concentrations were in the same range as those used in in vivo preparations in previous studies. Respiratory variables, i.e. respiratory frequency (breaths/min), the inspiratory $\left(\mathrm{T}_{\mathrm{I}}\right)$ and expiratory $\left(\mathrm{T}_{\mathrm{E}}\right)$ duration, as well as the peak amplitude of the rectified, integrated phrenic nerve activity (measured in arbitrary units, normalized to control), were measured in the period immediately preceding each trial, at the time when the maximum response to drug microinjections occurred and during the recovery period (see Mutolo et al., 2002, 2005; Bongianni et al., 2008, 2010; Pantaleo et al., 2011; Iovino et al., 2019; Cinelli et al., 2020). The respiratory effects observed in response to microinjections of several neuroactive agents into this region allowed us to characterize the rabbit preBötC. We believe that a better understanding of the mammalian respiratory CPG could be enhanced by comparative studies. However, we must take into account that previous results in rodents have been obtained from much reduced preparations, largely in vitro slice preparations, and this might lead to conclusions very different from those that can be drawn from results achieved under more intact respiratory network conditions in in vivo or in situ perfused brainstem preparations.

\section{Glutamatergic mechanisms}

Several lines of evidence indicate that glutamatergic transmission is essential for respiratory rhythmogenesis within the preBötC (Greer et al., 1991; Wallen-Mackenzie et al., 2006, 2010; Cook-Snyder et al., 2019; for review see Del Negro et al., 2018; Ramirez et al., 2016; Ramirez and Baertsch, 2018). Different hypotheses have been suggested to explain the mechanism underlying preBötC rhythmogenesis, from models based on pacemaker neurons to simple circuits dependent on inhibition (for review see Del Negro et al., 2018). One recent proposal is that the rhythm may be an emergent property of the preBötC microcircuit in which inspiratory bursts arise when and only when the preBötC rhythmogenic subpopulation strongly synchronizes to drive output neurons (Ashhad and Feldman, 2020). This hypothesis was first advanced by Shao et al. (2006) who showed rhythmic bursting in a simulated neural network where slow processes (intrinsic ion channel properties or synaptic mechanisms) do not exist, but rhythmic bursting is critically dependent on the connectivity of the network.

We showed that endogenously released excitatory amino acids (EAAs) play an important role in the control of both the intensity and frequency of inspiratory activity within the preBötC of the rabbit. However, a similar function of EAAs was found also within the BötC (Mutolo et al., 2005). We provided evidence that blockade of both N-methyl-D-aspartic acid (NMDA) and non-NMDA receptors by 
A a1

PA

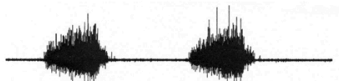

NA

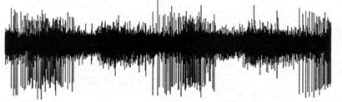

NA

a2

PA

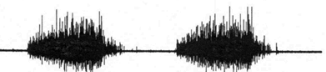

a4

PA

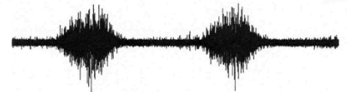

NA

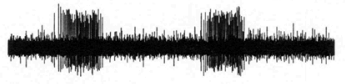

a5

PA

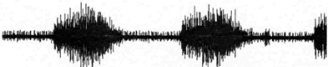

NA

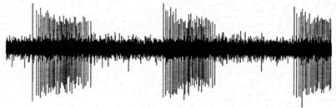

a3

PA

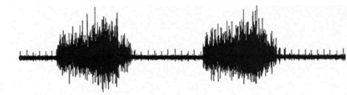

NA

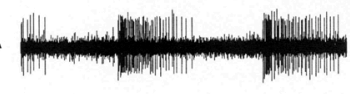

a6

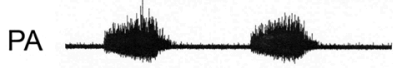

NA

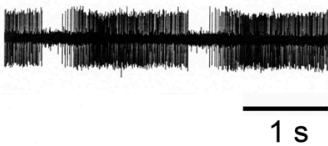

Fig. 1. Criteria for the localization of the preBötzinger complex. A: extracellular recordings of respiration-related neurons encountered in the preBötC showing different discharge patterns. Traces are phrenic nerve activity (PA) and extracellular neuronal activity (NA). a1: mix of inspiratory and expiratory neuronal activities. a2: expiratory augmenting discharge pattern. a3: expiratory decrementing (postinspiratory) discharge pattern. a4: inspiratory augmenting discharge pattern. a5: discharge pattern of an expiratory-inspiratory phasespanning neuron. a6: discharge pattern of an inspiratory-expiratory phase-spanning neuron. B: characteristic tachypneic effects in response to a unilateral microinjection of $20 \mathrm{mM}$ D, Lhomocysteic acid (DLH) into the preBötC. PA, phrenic nerve activity. C (left): diagrammatic representation of the dorsal view of the rabbit medulla oblongata illustrating some of the main components of the respiratory network. AP, area postrema; BötC, Bötzinger complex; cVRG, caudal ventral respiratory group; DRG, dorsal respiratory group; iVRG, inspiratory ventral respiratory group; preBötC, preBötzinger complex. C (right): photomicrograph of a coronal section of the medulla oblongata at the level indicated by the dashed line (about $2.5 \mathrm{~mm}$ rostral to the obex) showing an example of the location of fluorescent microspheres added to neuroactive agents and microinjected into the preBötC. This region is located ventro-medial to the rostral portion of the nucleus ambiguus. The histological section is counterstained with Cresyl violet. Light-field and fluorescent photomicrographs have been superimposed. NA, nucleus ambiguus; NOI, nucleus olivaris inferior; NTS, nucleus tractus solitarii; NV, nucleus tractus spinalis nervi trigemini; $\mathrm{P}$, tractus pyramidalis. Modified from Mutolo et al. (2002) and Cinelli et al. (2020).

$10 \mathrm{~s}$
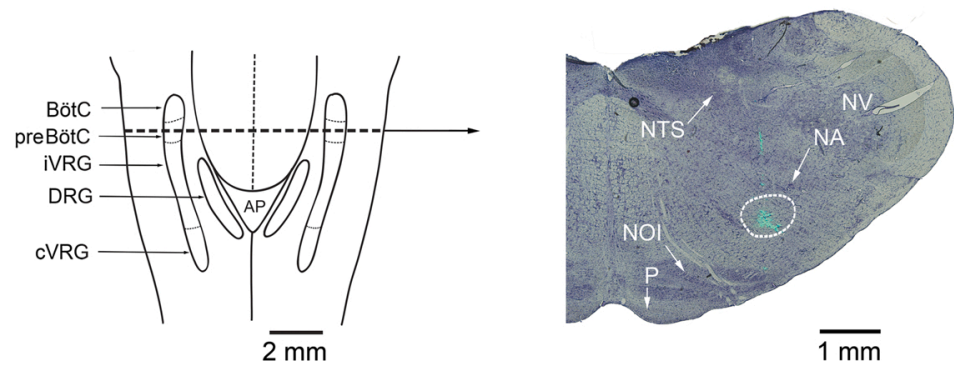

$20 \mathrm{mM}$ DLH

kynurenic acid within these two subregions induces respiratory responses (Fig. 2A) characterized by irregular, low-amplitude, high-frequency oscillations and in the BötC also by the development of tonic phrenic activity in the absence of any rhythmicity (tonic apnea). The results obtained with microinjections of the selective NMDA (D-AP5) or non-NMDA (CNQX) receptor antagonists indicated that the removal of the EAA-mediated excitatory inputs to the BötC and the preBötC produced severe reduction of the respiratory output similar to kainic acid lesions (Mutolo et al., 2002), and that NMDA receptors mediated the majority of excitatory inputs (Mutolo et al., 2005; Fig. 2B-D). This was confirmed in another in vivo study using a decerebrate rabbit model (Cook-Snyder et al., 2019). Prior studies in neonatal rat brainstem or slice preparations had found that glutamatergic inputs to the preBötC were solely mediated by non-NMDA receptors (Greer et al., 1991; Funk et al., 1993; Ge and Feldman, 1998; Morgado-Valle and Feldman, 2007). The importance of NMDA receptors may be specific for rabbits; alternatively, the difference may be due to the much higher level of excitatory drive to preBötC neurons present in in vivo preparations. Cook-Snyder et al. (2019) observed that sequential injection of D-AP5 and NBQX into the preBötC completely eliminated respiratory activity. Multiple studies in other species also showed that lesioning of the preBötC led to cessation of respiratory activity (Wenninger et al., 2004b;
McKay et al., 2005; Doi and Ramirez, 2008). In contrast, Mutolo et al. (2005) found that while injection of EAA receptor antagonists into the preBötC and BötC resulted in ineffective (life-threatening) respiratory patterns, the greater effect was obtained in the BötC. These effects were attributed to a desynchronization of the respiratory network leading to decreases in peak phrenic amplitude and increases of respiratory frequency (see Mutolo et al., 2005 also for further Refs.) as well as to tonic apnea possibly representing a level of desynchronization beyond which respiratory rhythm is absent. In agreement with other findings (Rybak et al., 2007, 2008), the results suggest that both these VRG subregions are important components of the rabbit respiratory CPG.

\section{GABAergic and glycinergic mechanisms}

The role of inhibitory neurotransmission in the generation of the breathing pattern was investigated by blocking $\mathrm{GABA}_{\mathrm{A}}, \mathrm{GABA}_{\mathrm{B}}$ and glycine receptors within the preBötC (Bongianni et al., 2010; see also Iovino et al., 2019). Bicuculline microinjections caused a pattern of breathing characterized by an overall decrease in respiratory frequency and the presence of two alternating different levels of peak phrenic activity (Fig. $3 \mathrm{~A}$ and $\mathrm{B}$ ). In contrast, the blockade of $\mathrm{GABA}_{\mathrm{B}}$ receptors did not alter respiratory activity. The finding that strychnine 
A

\section{Bötzinger complex}

IPA

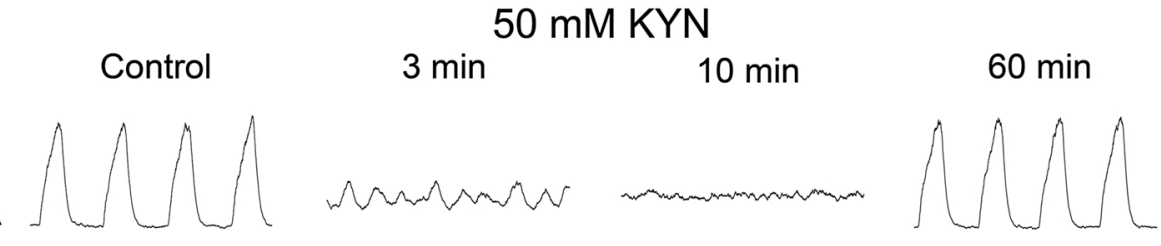

preBötzinger complex

$50 \mathrm{mM} \mathrm{KYN}$

Control $\quad 3 \mathrm{~min} \quad 10 \mathrm{~min} \quad 45 \mathrm{~min}$

IPA
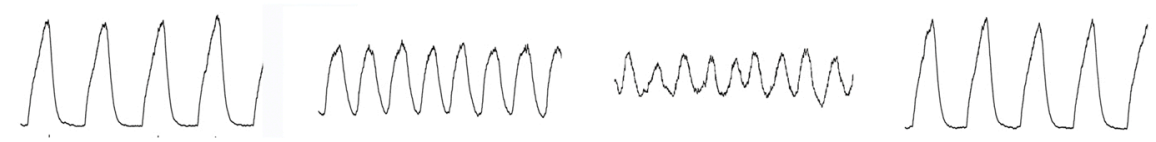

$\mathrm{B}$

preBötzinger complex

$20 \mathrm{mM}$ D-AP5

Control

IPA

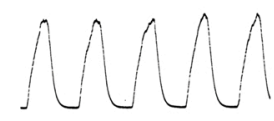

$2 \min$

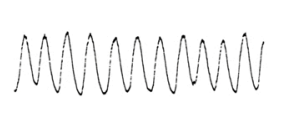

$10 \mathrm{~min}$

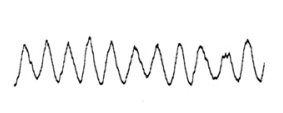

$45 \min$

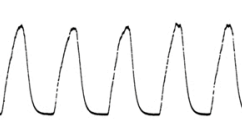

Fig. 2. Microinjections of excitatory amino acid receptor antagonists. A: respiratory responses caused by bilateral microinjections of $50 \mathrm{mM}$ kynurenic acid (KYN) into the Bötzinger complex and the preBötzinger complex. B: respiratory effects induced by bilateral microinjections of D-AP5 and CNQX at $20 \mathrm{mM}$ into the preBötzinger complex. In A and B recordings of integrated phrenic nerve activity (IPA) under control conditions and at different times after the completion of the injections are shown. C: histograms illustrating changes in respiratory frequency (RF) and peak phrenic activity (PPA) elicited by $20 \mathrm{mM}$ D-AP5 $(n=9)$ and $20 \mathrm{mM}$ CNQX $(n=9) 2$ min after the completion of the injections. Values are means \pm SEM. ${ }^{*} P<0.05$ compared with controls. D: representative coronal sections of the medulla oblongata of the rabbit showing the distribution of sites where microinjections of $20 \mathrm{mM}$ D-AP5 or CNQX were performed into the preBötzinger complex. Outlines of the maps derive from selected sections of one histological preparation (camera lucida redrawing). The atlas of Shek et al. (1986) was used for comparison. The distance in $\mathrm{mm}$ from the rostral margin of the area postrema (obex) is indicated on the left of each section. NA, nucleus ambiguus; NOI, nucleus olivaris inferior; NTS, nucleus tractus solitarii; $\mathrm{NV}$, nucleus tractus spinalis nervi trigemini; $\mathrm{P}$, tractus pyramidalis. Data and recordings adapted from Mutolo et al. (2005).

\section{$20 \mathrm{mM} C N Q X$}

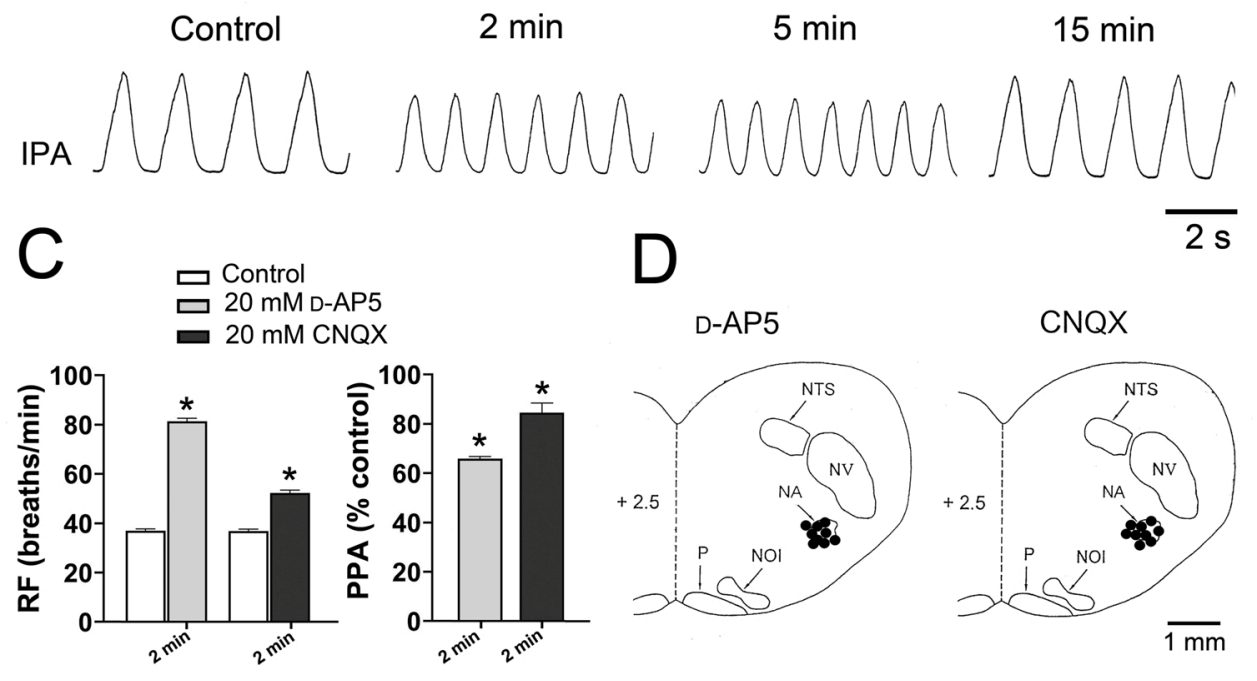

microinjections caused increases in respiratory frequency and decreases in peak phrenic amplitude (Fig. 3A and B) is consistent with previous findings (Pierrefiche et al., 1998) and suggests that glycinergic inputs converge on elements of the respiratory CPG. Thus, in the rabbit preBötC a potent inhibitory control is present, mainly mediated by the activation of $\mathrm{GABA}_{\mathrm{A}}$ receptors. As previously discussed (Bongianni et al., 2010), these inhibitory inputs may arise from several sources including the preBötC itself, the BötC, the NTS and the PB/KF nuclei.

The role of inhibitory neurons in the respiratory network is a matter of ongoing debate and the question of whether inhibitory neurons are critical for the generation of the respiratory rhythm is controversial (Baertsch et al., 2018, 2019, also for further Refs.). Although the blockade of both $\mathrm{GABA}_{\mathrm{A}}$ and glycine receptors does not stop the breathing rhythm, these inhibitory amino acids provide important contributions to the respiratory pattern formation. Inhibitory transmission during both inspiratory and expiratory phases of respiration may play a critical role in the control of both amplitude and frequency of the respiratory output (Menuet et al., 2020; Hülsmann et al., 2021). In fact, optogenetic studies performed in in vivo and in situ rodent preparations demonstrate that photoinhibition of preBötC neurons applied during inspiration stops the phrenic burst and triggers postinspiration, while during expiration prolongs the expiratory duration (Menuet et al., 2020). In addition, optogenetic activation of inhibitory preBötC neurons mediates phase-switching through the inhibition of excitatory 
A

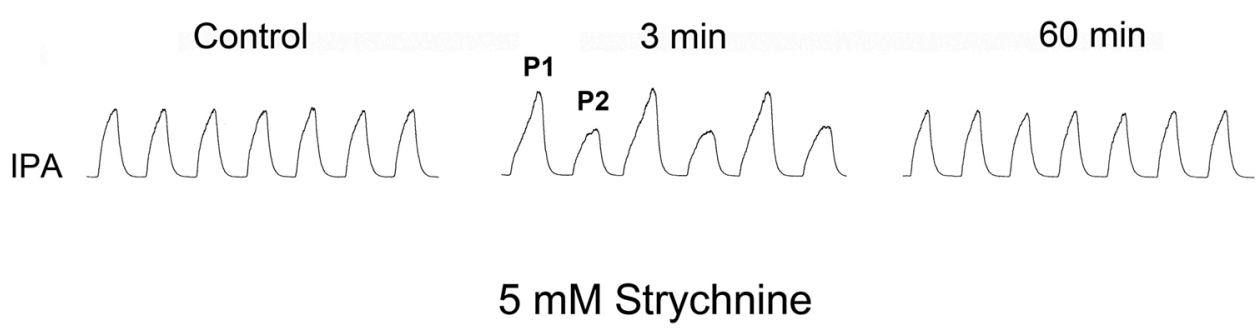

Control

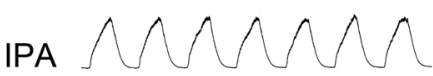

$3 \min$

$60 \min$
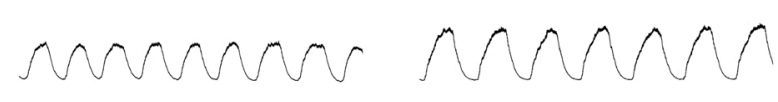

B

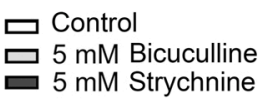

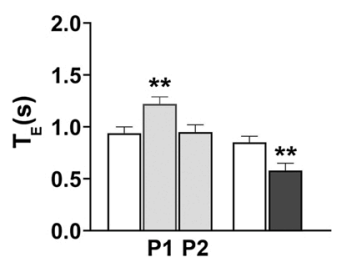

P1 P2

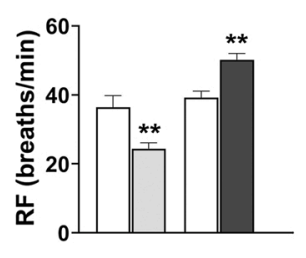

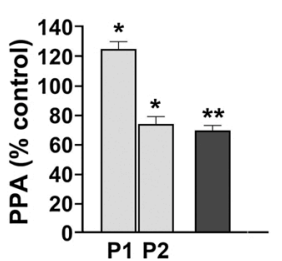

Fig. 3. Respiratory responses induced by the blockade of $\mathrm{GABA}_{\mathrm{A}}$ and glycine receptors within the preBötzinger complex. A: Effects caused by bilateral microinjections of $5 \mathrm{mM}$ bicuculline and $5 \mathrm{mM}$ strychnine under control conditions and at different times after the completion of the injections. Bicuculline elicited an overall decrease in respiratory frequency associated with the appearance of two alternating different levels of peak phrenic activity. Phrenic bursts with peak amplitude higher than control are indicated as P1 and those with peak amplitude lower than control as P2. Strychnine caused increases in respiratory frequency and decreases in peak phrenic amplitude. Traces are integrated phrenic nerve activity (IPA) under control conditions and at different times after the completion of the injections. B: histograms illustrating changes in inspiratory duration $\left(\mathrm{T}_{\mathrm{I}}\right)$, expiratory duration $\left(\mathrm{T}_{\mathrm{E}}\right)$, respiratory frequency (RF) and peak phrenic activity (PPA) in response to bicuculline $(n=5)$ and strychnine $(n=5) 3$ min after the completion of the injections. Values are means \pm SEM. ${ }^{*} P<0.05 ; * * P<0.01 ; * * * P<$ 0.001 compared with controls. Data and recordings adapted from Bongianni et al. (2010). rhythmogenic neurons in in vivo mice (Hülsmann et al., 2021). At variance with previous experiments in the rodent preBötC (e.g. Kam et al., 2013; Janczewski et al., 2013; Sherman et al., 2015; Cui et al., 2016), these latter studies demonstrate that optogenetic activation of this region can modulate either inspiratory or expiratory activity.

Consistent with the changes in the breathing pattern induced by $\mathrm{GABA}_{\mathrm{A}}$ receptor blockade in our experiments, a recent study by Baertsch et al. (2018) in mice performed in vivo and in vitro (medullary slices) demonstrates that GABAergic inhibition decreases the inspiratory duration and reduces the refractory period, thus allowing an increase in respiratory frequency. Removing GABAergic and glycinergic inhibition increases the duration and the amplitude of inspiratory bursts, prolongs the refractory period leading to a decrease in respiratory frequency. A limited increase in the duration of the inspiratory burst after blockade of GABAergic inhibition has also been reported by Cui et al. (2016) in in vivo transgenic mice. Interestingly, the results by Baertsch and collaborators (2019) using in vivo and in vitro (horizontal medullary slices) mice preparations show that GABAergic inhibition increases the frequency of sighs. The alternating pattern of inspiratory activity observed in our experiments after $\mathrm{GABA}_{\mathrm{A}}$ receptor blockade seems to imply an impairment of the inspiratory off-switch mechanism affecting the higher bursts. Inhibitory inputs to the preBötC play a role in the inspiratory off-switch (see e.g., Rybak et al., 2008). Thus, the removal of inhibitory inputs may result in larger (fictive) tidal volume breaths, which resemble augmented breaths. Similarly, $\mathrm{GABA}_{\mathrm{A}}$ receptor blockade within the preBötC in adult cats can produce augmented breaths (Solomon, 2000; see Bongianni et al., 2010 for further details)

Interestingly, a recent study (Furuya et al., 2021) challenges the view that glycinergic mechanisms within the preBötC underlie respiratory pattern formation. In fact, it provides evidence in the in situ working-heart brainstem preparations of rats that while systemic pharmacological perturbation of glycinergic neurotransmission potently modulates the respiratory frequency, local perturbation of glycinergic neurotransmission in the preBötC has almost no effect on the respiratory frequency or phase durations. Interestingly, inhibitory mechanisms have been shown to play a role in the serotoninergic modulation of preBötC neurons (see also below under section 4.4 Serotonin).

\section{Modulatory role of some neuroactive agents}

Neuromodulation involved in the process of respiratory rhythm and pattern generation is very complex and comprises several neuromodulators and different areas of the central nervous system (see e.g. Doi and Ramirez, 2008; Ramirez et al., 2016; Ramirez and Baertsch, 2018). Neuromodulators are implicated in the regulation of frequency and amplitude of respiratory activity and in the adaptive control of the respiratory network during different breathing behaviours.

\subsection{Neurokinins}

It is well known that substance $\mathrm{P}(\mathrm{SP})$ microinjected into the preBötC causes increases in respiratory frequency in rodent in vivo, in situ and in vitro (medullary slices) preparations (Gray et al., 1999, 2001; Wang et al., 2002; Peña and Ramirez, 2004; McKay et al., 2005; Fong and Potts, 2006; Hayes and Del Negro, 2007). Furthermore, respiratory rhythm is dramatically altered after the ablation of $\mathrm{NK}_{1}$ receptor-expressing neurons in the preBötC of rats and goats (Gray et al., 2001; Wang et al., 2002; Wenninger et al., 2004a; McKay et al., 2005; for review see Del Negro et al., 2018). On the other hand, it has been suggested that SP provokes depolarization of preBötC neurons through an action not only on $\mathrm{NK}_{1}$, but also neurokinin-2 $\left(\mathrm{NK}_{2}\right)$ and neurokinin-3 $\left(\mathrm{NK}_{3}\right)$ receptors (Peña and Ramirez, 2004; Hayes and Del Negro, 2007). We observed that microinjections of SP into the rabbit preBötC induced increases in respiratory frequency (Bongianni et al., 2008). However, these effects were mimicked by the activation of the $\mathrm{NK}_{2}$ receptor agonist neurokinin $\mathrm{A}$ and the $\mathrm{NK}_{3}$ receptor agonist senktide, but 
not by the application of two different $\mathrm{NK}_{1}$ receptor agonists, i.e. [Sar', $\operatorname{Met}\left(\mathrm{O}_{2}\right)^{11}$ ]-SP and GR73632 (Fig. 4A and B). Injection of the $\mathrm{NK}_{2}$ receptor antagonist MEN10376 into the preBötC decreased peak phrenic activity. In addition, the $\mathrm{NK}_{1}$ receptor antagonist CP-99,994 did not antagonize the effects of SP. Although $\mathrm{NK}_{1}$ receptors do not seem to be expressed in the preBötC of the rabbit, they are present also in this animal. In fact, $\mathrm{NK}_{1}$ receptor agonists injected into the fourth ventricle increased respiratory frequency, thus indicating that $\mathrm{NK}_{1}$ receptors are located on neurons involved in respiratory regulation, but different from those located within the preBötC. These findings could be related to species-dependent differences in the presence and distribution of the $\mathrm{NK}_{1}$ receptors (Pennefather et al., 2004; Bongianni et al., 2008; for review see Severini et al., 2002). The main conclusion of our study is that $\mathrm{NK}_{2}$ and $\mathrm{NK}_{3}$ receptors, but not $\mathrm{NK}_{1}$ receptors exert an important modulation of inspiratory activity in the rabbit preBötC (for further details and Refs. see Bongianni et al., 2008).
A

\section{$5 \mathrm{mM} \mathrm{SP}$}

Control

$1 \min$

IPA

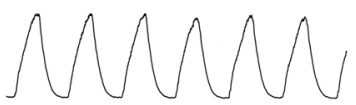

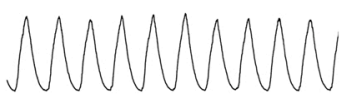

$60 \mathrm{~min}$

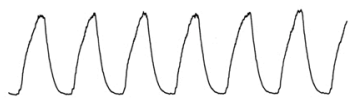

\section{$5 \mathrm{mM}\left[\operatorname{Sar}{ }^{9}, \operatorname{Met}\left(\mathrm{O}_{2}\right)^{11}\right]-\mathrm{SP}$}

Control

IPA

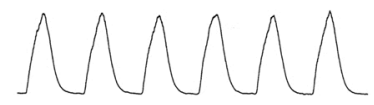

$1 \mathrm{~min}$

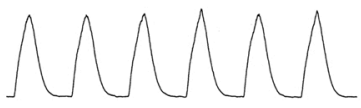

$5 \mathrm{mM}$ Neurokinin A
Fig. 4. Respiratory responses to bilateral microinjections of neurokinin receptor agonists into the preBötzinger complex. A: respiratory effects of $5 \mathrm{mM}$ SP, $5 \mathrm{mM}\left[\operatorname{Sar}^{9}, \operatorname{Met}\left(\mathrm{O}_{2}\right)^{11}\right]$-SP, $5 \mathrm{mM}$ neurokinin $\mathrm{A}$ and $5 \mathrm{mM}$ senktide are shown. Traces are integrated phrenic nerve activity (IPA) under control conditions and at different times after the completion of the injections. Note that the $\mathrm{NK}_{1}$ receptor agonist $\left[\mathrm{Sar}^{9}, \operatorname{Met}\left(\mathrm{O}_{2}\right)^{11}\right]$-SP did not alter respiratory activity. B: histograms illustrating changes in inspiratory duration $\left(\mathrm{T}_{\mathrm{I}}\right)$, expiratory duration $\left(\mathrm{T}_{\mathrm{E}}\right)$, respiratory frequency $(\mathrm{RF})$ and peak phrenic activity (PPA) induced by each neurokinin receptor agonist $1 \mathrm{~min}$ after the completion of the microinjections ( $n=6$ for each agonist). Values are means \pm SEM. ${ }^{*} P<0.05$ compared with controls. Data and recordings adapted from Bongianni et al. (2008).
Control

IPA

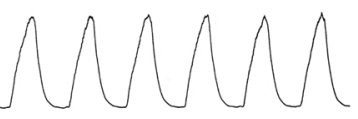

$1 \min$

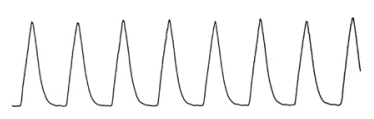

\section{5 mM Senktide}

Control

IPA

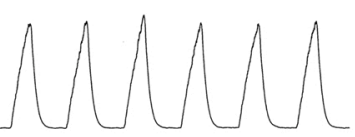

$1 \min$

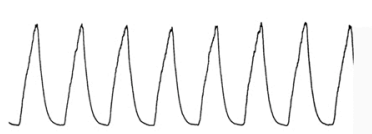

$60 \min$

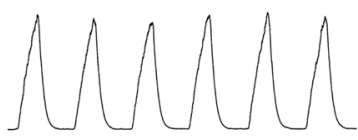

B
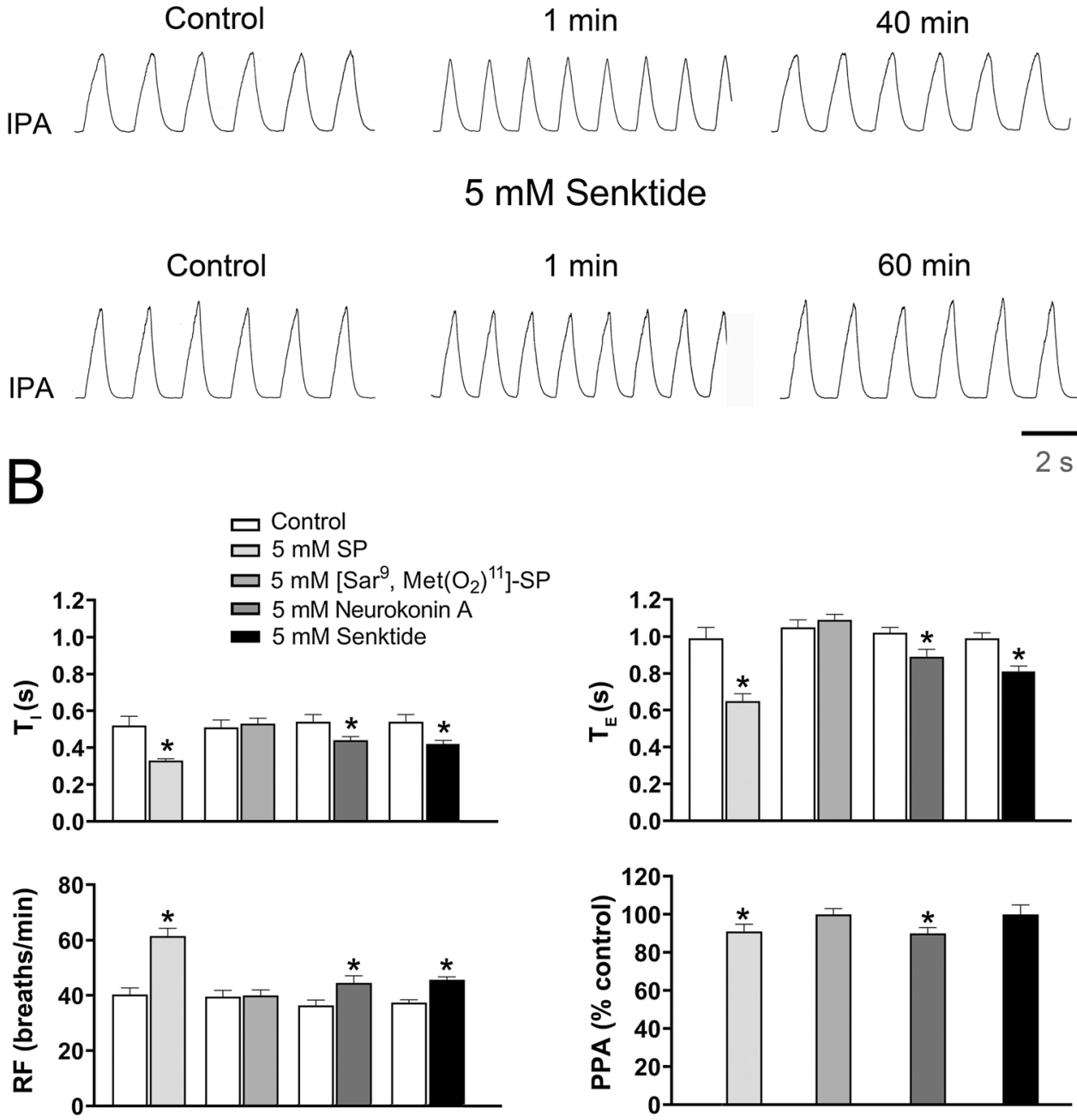


\subsection{Somatostatin}

Excitatory glutamatergic neurons within the preBötC express high levels of $\mathrm{NK}_{1}$ receptors and display colocalization of the peptide somatostatin (SST). They play an essential role in the generation of the breathing pattern (Tan et al., 2008; Cui et al., 2016; Ashhad and Feldman, 2020; for review see Del Negro et al., 2018). Bilateral silencing of SST neurons causes persistent apnea in awake adult rats (Tan et al., 2008), whereas photostimulation of inspiratory SST neurons in early inspiration induces an augmented inspiratory burst in transgenic adult mice expressing channelrhodopsin 2 in $\mathrm{SST}^{+}$neurons, suggesting that they primarily act to pattern respiratory motor output (Cui et al., 2016).

Results from several studies indicate that SST functions as an inhibitory neuromodulator. Llona et al. (2004) showed that SST depresses inspiratory burst frequency and amplitude in an isolated brainstem-spinal cord preparation from newborn mice. Burke et al. (2010) reported in anesthetized rats that SST microinjections into the preBötC caused depressant effects on respiratory activity or apnea, whereas those performed into the BötC induced apneustic effects accompanied by the suppression of postinspiratory activity. Furthermore, microperfusion of SST into the preBötC decreases respiratory frequency in anesthetized rats via $\mathrm{SST}_{2}$ receptors (Montandon et al., 2016a). Similarly, it has been reported in neonatal mice that SST depresses respiratory activity and that the blockade of $\mathrm{SST}_{2}$ receptors increases respiratory frequency, pointing to a tonic release of this peptide in the preBötC (Ramirez-Jarquin et al., 2012). These findings are consistent with the results obtained by Gray et al. (2010) in an en bloc preparation from neonatal mice, where SST induced step-like decreases in inspiratory frequency due to "quantal slowing" (see e.g. Mellen et al., 2003).

We provided evidence (Pantaleo et al., 2011) that SST receptors are involved in the modulation of respiratory activity in the adult rabbit through an action on BötC and preBötC neurons, while they are not present within the iVRG. In particular, SST microinjections into the BötC reduced respiratory frequency and the rate of rise of phrenic activity without changes in its peak amplitude. Surprisingly, SST microinjected into the preBötC caused increases in respiratory frequency (due to decreases in both $\mathrm{T}_{\mathrm{I}}$ and $\mathrm{T}_{\mathrm{E}}$ ) and rate of rise of inspiratory activity accompanied by small, but significant decreases in peak phrenic amplitude. Cyclosomatostatin, a non-specific SST receptor antagonist, microinjected into the preBötC decreased respiratory frequency (due to increases in both $\mathrm{T}_{\mathrm{I}}$ and $\mathrm{T}_{\mathrm{E}}$ ) and increased peak amplitude and rate of rise of phrenic nerve activity, indicating that endogenously released SST within this region modulates basal breathing. Our results are at variance with the depressant effects on respiration (bradypnoea or even apnea) observed in previous studies performed on rats and mice (Burke et al., 2010; Ramirez-Jarquin et al., 2012; Montandon et al., 2016a). However, respiratory effects caused by SST microinjected into the BötC display similarities with those reported by Burke et al. (2010). We do not know the reasons of these discrepancies; however, we hypothesized (Pantaleo et al., 2011) that differences in the animal species and in the pattern of expression of SST receptors within the respiratory network may have played a role. Furthermore, we proposed that the respiratory responses caused by the SST microinjections could be ascribed to disinhibition phenomena evoked by the activation of SST receptors on inhibitory neurons at pre- or postsynaptic levels. It is well known that SST has inhibitory effects on neuronal excitability and modulates the release of several neurotransmitters (Llona and Eugenin, 2005; Spary et al., 2008). In addition, a presynaptic action of this peptide on GABAergic neurotransmission has been observed (Leresche et al., 2000; Spary et al., 2008). Finally, SST could have removed an inhibitory action on the release of excitatory neurotransmitters, such as glutamate and NKs (Bongianni et al., 1997, 2008; Mutolo et al., 2005; see Pantaleo et al., 2011 for further details and Refs.).

\subsection{Opioids}

A subpopulation of preBötC glutamatergic neurons characterized by the expression of $\mathrm{NK}_{1}$ receptors contains $\mu$-opioid receptors (MORs; Smith et al., 1991; Gray et al., 1999, 2001; McKay et al., 2005; Tan et al., 2008; for review see Feldman et al., 2003; Del Negro et al., 2018). It is well known that the preBötC contributes to MOR-induced respiratory depression in medullary slices from neonatal rodents (Gray et al., 1999; Sun et al., 2019) as well as in anesthetized or conscious adult rats (Montandon et al., 2011, 2016b) and in anesthetized, paralyzed and artificially ventilated adult rats (Qi et al., 2017). However, conflicting results have been obtained at least in adult animals (Lonergan et al., 2003; Mustapic et al., 2010; Prkic et al., 2012; Stucke et al., 2015; Miller et al., 2017). In particular, consistent increases in respiratory frequency accompanied by relatively small decreases in peak phrenic amplitude have been described after MOR activation within the preBötC in adult rats (Lonergan et al., 2003) and dogs (Mustapic et al., 2010), while no changes in the eupneic breathing have been reported after DAMGO microinjections into the preBötC of awake adult goats (Krause et al., 2009). A recent study performed in young and adult rabbits investigating the respiratory responses induced by DAMGO microinjections into the preBötC as well as those due to the systemic administration of the opioid agonist remifentanil has led to the conclusion that systemic opioids may also affect respiratory components outside the preBötC (Stucke et al., 2015). Accordingly, it has been reported that in in vivo preparations of both rabbits and mice other respiration-related regions critical for the maintenance of the eupneic pattern of breathing, such as the pontine $\mathrm{PB} / \mathrm{KF}$ nuclei, have a role in the opioid-induced respiratory depression (Levitt et al., 2015; Miller et al., 2017; Bachmutsky et al., 2020; Varga et al., 2020).

We investigated the role of the preBötC, BötC and iVRG in the mediation of opioid-induced respiratory depression in adult rabbits (Cinelli et al., 2020). Dose-dependent effects on respiratory activity, counteracted by naloxone, were induced by MOR-activation in all the investigated regions. In detail, DAMGO microinjections into the preBötC and the BötC evoked reductions in peak phrenic amplitude associated with tonic activity and irregular (ataxic) patterns of breathing, that were more pronounced in the preBötC (Fig. 5). Similar microinjections into the iVRG induced decreases in frequency and amplitude of phrenic bursts and apnea. DAMGO concentrations of at least $0.1 \mathrm{mM}$ were required to achieve a significant respiratory effect, i.e., higher than tissue concentrations during systemic opioid administration, which were reported in the low nanomolar range (Michelsen et al., 1996). Given our small injection volumes, these barrel concentrations were necessary to achieve an effective drug concentration at a sufficient number of preBötC neurons. For an extensive discussion of microinjection procedures and drug diffusion see Lipski et al. (1988); Nicholson and Sykova (1998); Bongianni et al. (2002, 2008, 2010), Iovino et al. (2019); Cinelli et al. (2020).

Our results support the notion that the preBötC neurons that are essential for respiratory rate and rhythm generation are susceptible to opioid-induced depression. Furthermore, they support the view that respiratory pattern formation engages distributed neuronal populations within the different brainstem respiratory compartments (see e.g. Von Euler, 1997; Mutolo et al., 2002; Smith et al., 2007; Jones and Dutschmann, 2016; Dhingra et al., 2019, 2020 also for further Refs.).

\subsection{Serotonin}

Serotonin (5-HT) plays a key role in the modulation of the breathing pattern and, in particular, provides an excitatory drive to respiratory activity through an action on the preBötC (Hodges and Richerson, 2008, 2010; Hilaire et al., 2010). These respiratory effects have been reported mainly in studies on neonatal and juvenile in situ rodent preparations ( $e$. g. Al Zubaidy et al., 1996; Peña and Ramirez, 2002; Schwarzacher et al., 2002; Gunther et al., 2006; Ptak et al., 2009; Niebert et al., 2011; 
A

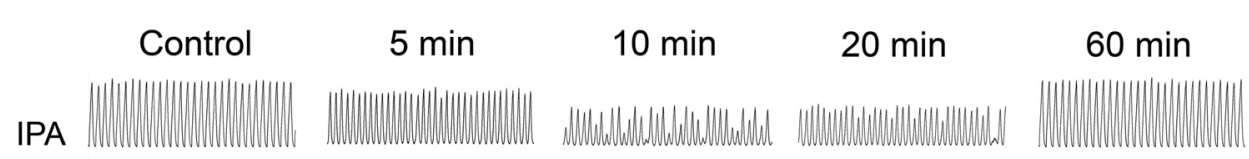

B

\section{$0.5 \mathrm{mM}$ DAMGO}

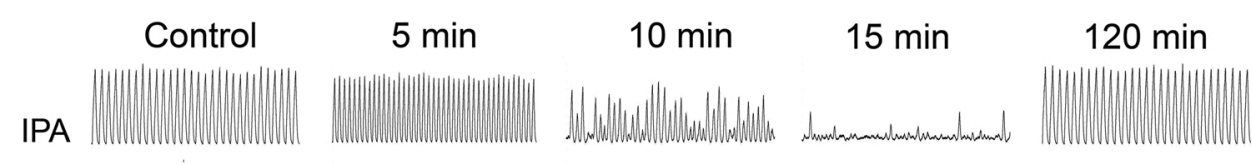

C

\section{$0.5 \mathrm{mM}$ DAMGO}

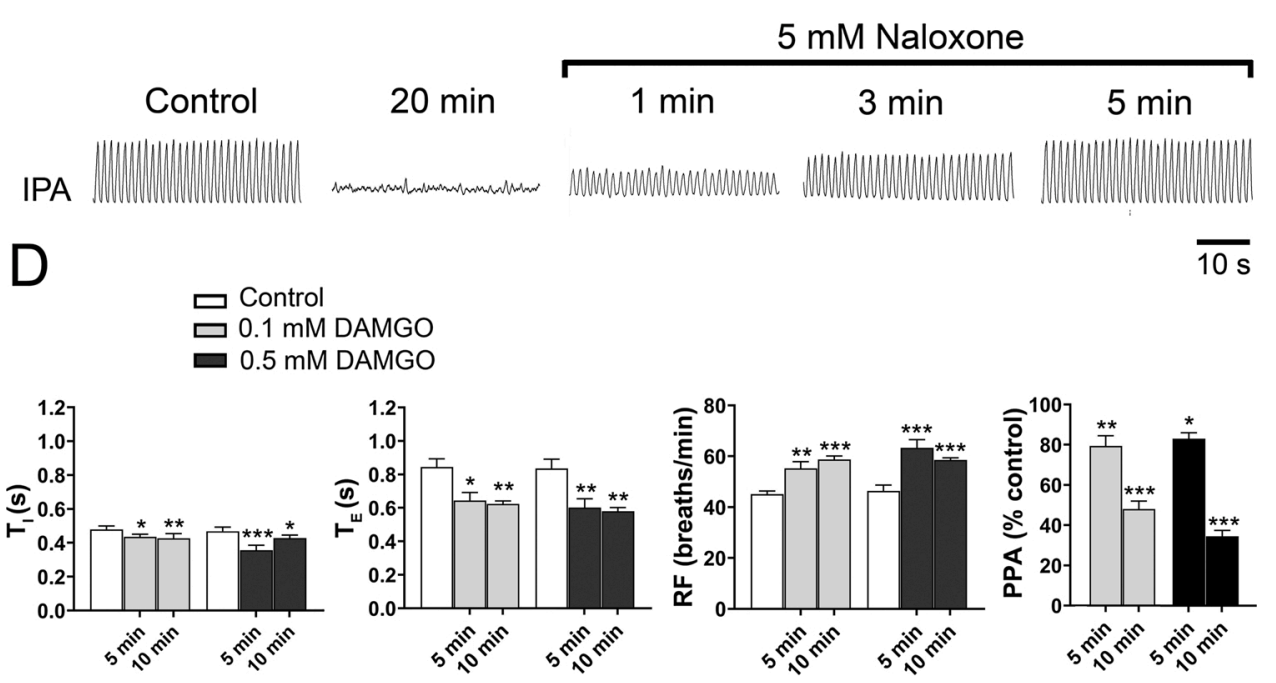

Fig. 5. Microinjections of the $\mu$-opioid receptor agonist DAMGO into the preBötzinger complex. Respiratory effects caused by bilateral microinjections of $0.1 \mathrm{mM}$ (A) and $0.5 \mathrm{mM}$ (B) DAMGO under control conditions and at different times after the completion of the injections. Note the development of tonic activity in response to DAMGO microinjections. C: reversion of the apneic effects observed about $20 \mathrm{~min}$ after $0.5 \mathrm{mM}$ DAMGO by bilateral microinjections of $5 \mathrm{mM}$ naloxone into the same sites. IPA, integrated phrenic nerve activity. D: histograms illustrating changes in inspiratory duration $\left(\mathrm{T}_{\mathrm{I}}\right)$, expiratory duration $\left(\mathrm{T}_{\mathrm{E}}\right)$, respiratory frequency (RF) and peak phrenic activity (PPA) after bilateral microinjections of $0.1 \mathrm{mM}$ $(n=5)$ and $0.5 \mathrm{mM}(n=5)$ DAMGO at selected times (5 and $10 \mathrm{~min}$ ) after the completion of the injections. Values are means \pm SEM. ${ }^{*} P<0.05$, ** $P<0.01$, *** $P<0.001$ compared with controls. Data and recordings adapted from Cinelli et al. (2020).
Corcoran et al., 2014; for review see Hilaire et al., 2010; Hodges and Richerson, 2010). Medullary serotoninergic neurons, mainly located in the brainstem raphe nuclei and parapyramidal regions, project extensively to the brainstem respiratory network, including the preBötC (Ptak et al., 2009; see also Hodges and Richerson, 2008; Paterson et al., 2009; Hilaire et al., 2010; Ramirez et al., 2013 also for further refs) and have been proposed as putative central chemoreceptors (Hodges and Richerson, 2008, 2010; Teran et al., 2014). Interestingly, a study by DePuy and colleagues (2011) demonstrated using optogenetics in anesthetized mice that stimulation of raphe obscurus serotoninergic neurons increased both respiratory and amplitude of the respiratory output and potentiated the respiratory response to $\mathrm{CO}_{2}$. However, DePuy et al. (2011) conclude that most probably these serotoninergic neurons are not central chemoreceptors since the respiratory response to optogenetic stimulation did not change under hypercapnic conditions. It is worth noting that ionotropic and metabotropic 5-HT receptors are widely expressed in the VRG and the tachypneic effects are obviously dependent on the activation of pre- and postsynaptic 5-HT receptors within the respiratory network (e.g. Hodges and Richerson, 2008, 2010; Nichols and Nichols, 2008; Dutschmann et al., 2009; Hilaire et al., 2010). Furthermore, 5-HT-mediated inhibition of inhibitory glycinergic neurons has been proposed to contribute to the tachypneic effects (e.g. Manzke et al., 2009; Shevtsova et al., 2011; Corcoran et al., 2014). According to all the above mentioned studies, the most frequently

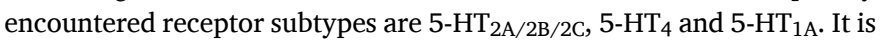
worth noting that some of these 5 -HT receptor subtypes are involved in the maintenance of the respiratory rhythm under basal conditions. For instance, previous studies on transverse brainstem slices of neonatal mice have shown that endogenously-released 5- $\mathrm{HT}$ acting on $5-\mathrm{HT}_{2 \mathrm{~A}}$ receptors contribute to the generation of the ongoing respiratory activity (Peña and Ramirez, 2002).

We have recently investigated the respiratory role of 5-HT within the preBötC and neighbouring respiration-related regions in adult rabbits (Iovino et al., 2019). 5-HT caused increases in respiratory frequency associated with reductions in peak phrenic amplitude only when microinjected into the preBötC (Fig. 6A) via the activation of $5-\mathrm{HT}_{1 \mathrm{~A}}$ and $5-\mathrm{HT}_{3}$ receptors (Fig. $6 \mathrm{~B}$ and $\mathrm{C}$ ). Surprisingly, the blockade of $5-\mathrm{HT}_{1 \mathrm{~A}}$ receptors by methysergide or the specific antagonist (S)-WAY 100,135 induced similar tachypneic effects (Fig. 6D), that were prevented by microinjections of the $5-\mathrm{HT}_{3}$ receptor antagonist ondansetron. The blockade of $5-\mathrm{HT}_{3}$ receptors per se did not alter respiration. Part of these results have also been reported as histograms in Fig. 6E. Furthermore, the effects evoked by the $5-\mathrm{HT}_{1 \mathrm{~A}}$ receptor agonist $8-\mathrm{OH}$-DPAT were prevented by $\mathrm{GABA}_{\mathrm{A}}$ receptor antagonist bicuculline microinjected into the preBötC, thus indicating an involvement of a GABAergic inhibitory circuit subserving 5-HT-mediated responses. This finding was also corroborated by immunohistochemical data showing that GABAergic immunoreactive structures are present in the preBötC and that $5-\mathrm{HT}_{1 \mathrm{~A}}$ receptors are widely expressed in association with the soma of GABAergic immunoreactive neurons (Fig. 7A1-3). It seems appropriate to consider that, in general, increases in respiratory rate may not represent an increase in the excitatory drive to excitatory CPG neurons, 
A

$5 \mathrm{mM} 5-\mathrm{HT}$

Control

IPA

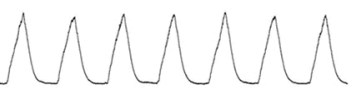

B

\section{$1 \mathrm{mM}$ 8-OH-DPAT}

Control

IPA

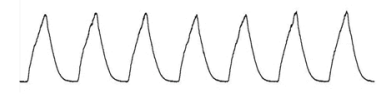

C

$5 \mathrm{mM}$ PBG

Control

IPA

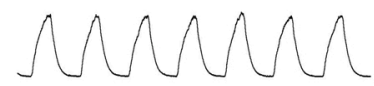

$D$
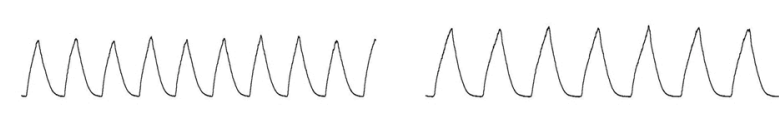

$10 \min$

$60 \min$
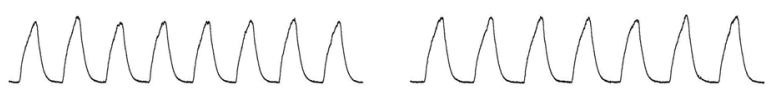

$5 \mathrm{mM}(\mathrm{S})$-WAY

Control

IPA

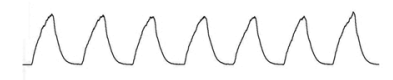

$10 \min$
Fig. 6. Microinjections of 5-HT receptor agonists and antagonist into the preBötzinger complex. Excitatory effects on phrenic activity in response to $5-\mathrm{HT}(\mathrm{A})$, the $5-\mathrm{HT}_{1 \mathrm{~A}}$ receptor agonist $8-\mathrm{OH}-\mathrm{DPAT}$ (B), the $5-\mathrm{HT}_{3}$ receptor agonist PBG (C) and the $5-\mathrm{HT}_{1 \mathrm{~A}}$ receptor antagonist (S)-WAY 100,135 (D). Traces are integrated phrenic nerve activity (IPA) under control conditions and at different times after the completion of the injections. E: histograms illustrating changes in inspiratory duration $\left(\mathrm{T}_{\mathrm{I}}\right)$, expiratory duration $\left(\mathrm{T}_{\mathrm{E}}\right)$, respiratory frequency (RF) and peak phrenic activity (PPA) elicited by the employed drugs $10 \mathrm{~min}$ after bilateral microinjections ( $n=6$ for each drug). Values are means \pm SEM. ${ }^{*} P<0.05, * * P<0.01, * * * P<$ 0.001 compared with controls. Data and recordings adapted from Iovino et al. (2019).

E
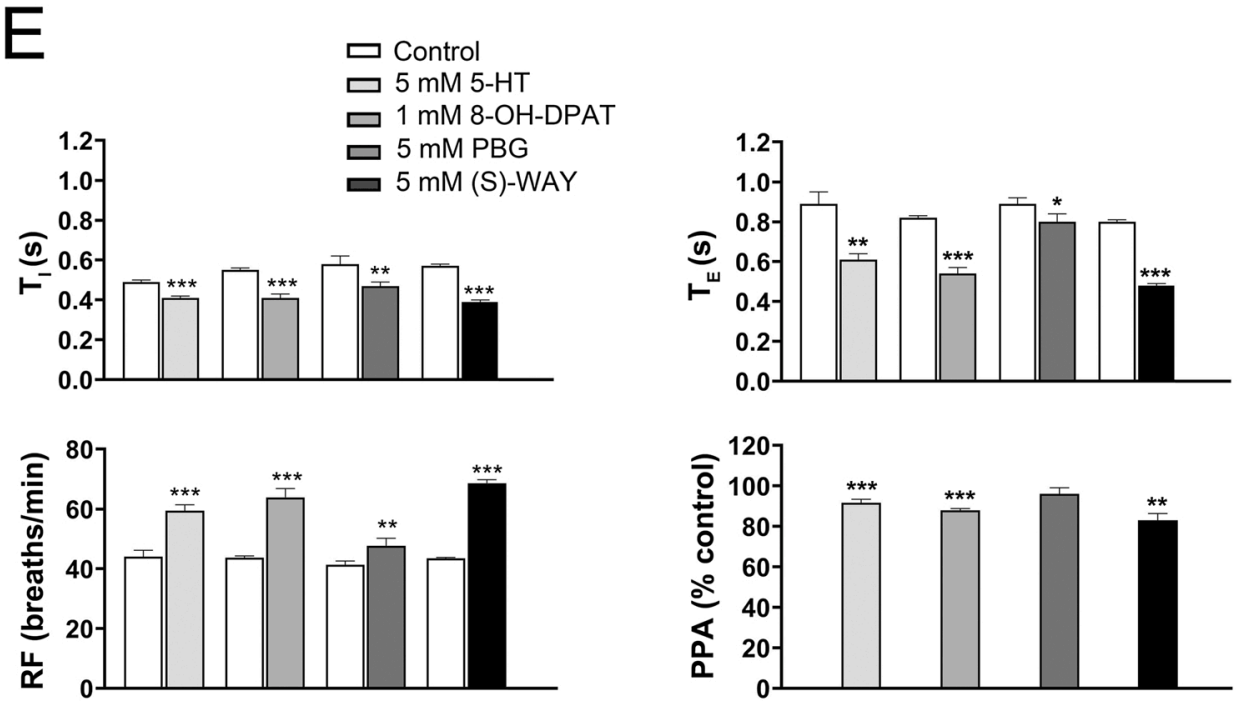

but may also be due to a decrease in excitatory inputs as well as to an increase in inhibitory inputs to reciprocally inhibitory CPG neurons, i.e. decreases in reciprocal inhibition or disinhibition, respectively. Two hypothetical 5-HT mechanisms responsible for the activation of preBötC excitatory neurons are shown in Fig. 7B. Under baseline conditions, the differential effects observed by applying specific $5-\mathrm{HT}_{3}$ and $5-\mathrm{HT}_{1 \mathrm{~A}}$ receptor antagonists suggest that only presynaptic $5-\mathrm{HT}_{1 \mathrm{~A}}$ receptors are endogenously activated and, in addition, that 5-HT release should be minimum, if any, since the ongoing respiratory activity is not altered by $5-\mathrm{HT}_{3}$ and $5-\mathrm{HT}_{1 \mathrm{~A}}$ receptor blockade. Obviously, these considerations are inferred from our results in the adult rabbit, where the other receptor subtypes do not seem to be present as shown by the lack of effects in response to their specific agonists (see Iovino et al., 2019). When serotoninergic mechanisms are activated, two different mechanisms may be engaged (Fig. 7B). The first involves the release of 5-HT regulated by $5-\mathrm{HT}_{1 \mathrm{~A}}$ receptor-mediated presynaptic inhibition and the activation of the ionotropic excitatory $5-\mathrm{HT}_{3}$ receptors located on preBötC excitatory neurons. The second mechanism concerns the release of 5-HT (possibly controlled by presynaptic $5-\mathrm{HT}_{1 \mathrm{~A}}$ receptors) and the activation of inhibitory postsynaptic $5-\mathrm{HT}_{1 \mathrm{~A}}$ receptors on a specific set of GABAergic cells innervating the preBötC excitatory neurons with consequent disinhibition phenomena. 

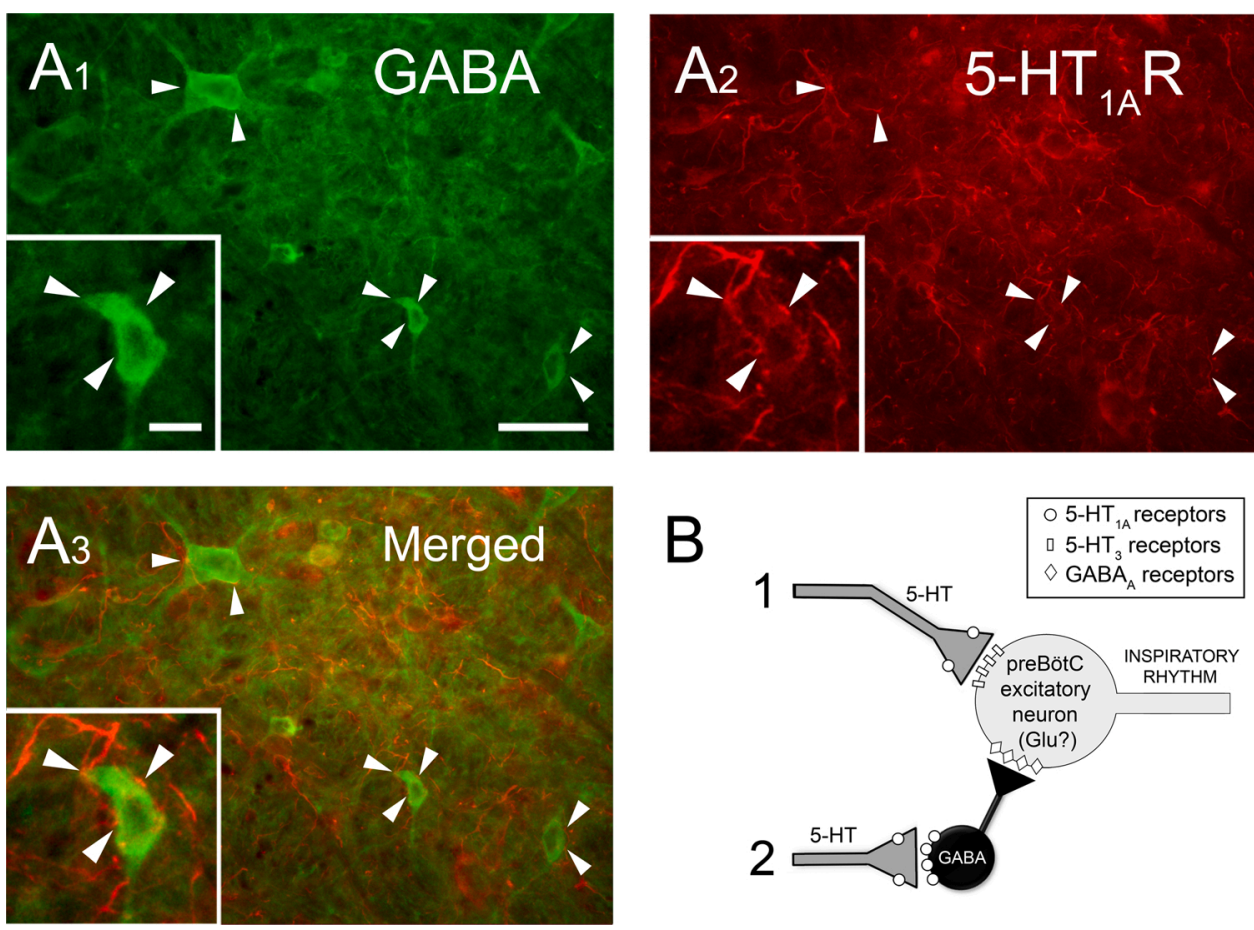

Fig. 7. Distribution of GABA and $5-\mathrm{HT}_{1 \mathrm{~A}}$ receptor immunoreactivity within the preBötzinger complex and the hypothetical mechanisms underlying 5-HT-induced respiratory responses. A1-3: Photomicrographs show GABAergic immunoreactive structures (A1, green signal), $5-\mathrm{HT}_{1 \mathrm{~A}}$ receptor binding sites (A2, red signal) and merged image (A3). Arrowheads point to $5-\mathrm{HT}_{1 \mathrm{~A}}$-immunoreactive structures located in close apposition to GABAergic neurons. The insets (higher magnification) indicate the location of some $5-\mathrm{HT}_{1 \mathrm{~A}^{-}}$ immunoreactive dots in close proximity to a single GABAergic neuron. Scale bars: A1-3, 50 $\mu \mathrm{m}$; insets, $10 \mu \mathrm{m}$. B: schematic drawing, inferred from the present results, representing two hypothetical 5-HT mechanisms responsible for the activation of preBötC excitatory neurons. The first mechanism (1) consists of the release of 5 -HT regulated by $5-\mathrm{HT}_{1 \mathrm{~A}}$ receptormediated presynaptic inhibition and the activation of the ionotropic excitatory $5-\mathrm{HT}_{3}$ receptors located on preBötC excitatory neurons. The second mechanism (2) involves the release of 5 -HT causing the activation of $5-\mathrm{HT}_{1 \mathrm{~A}}$ inhibitory receptors on a specific set of GABAergic cells innervating the preBötC excitatory neurons. Disinhibition of these inhibitory neurons leads to excitatory effects on respiration. Modified from Iovino et al. (2019).

\section{Concluding remarks}

The preBötC is a neural network containing a heterogeneous neuronal population crucial for respiratory rhythm generation and pattern formation. Much remains to be clearly assessed about the role of different subclasses of preBötC neurons and related microcircuits. This region is characterized by the presence of multiple neuronal receptors subserving the integration of many inputs to generate and modify the breathing behaviour. The results reported in this short review provide an overview of the main characteristics of neurochemical mechanisms operating within the preBötC of the rabbit. In agreement with the results obtained in different animal models, not only excitatory amino acids, but also GABA and glycine provide an important contribution to respiratory pattern formation.

Present findings, along with the results of some previous studies obtained in in vivo and in vitro experiments, confirm that SP microinjected into the preBötC region induces marked increases in respiratory activity. However, in the rabbit the respiratory responses were not due to the activation of $\mathrm{NK}_{1}$ receptors, but to that of $\mathrm{NK}_{2}$ and $\mathrm{NK}_{3}$ receptors. Although the results of several studies indicate that SST functions as an inhibitory neuromodulator of respiratory activity, we observed that SST microinjected into the preBötC increased the respiratory rate. Our findings in the adult rabbit also suggest further possible insights into the role of MORs in the genesis of opioid-related respiratory depression. We are confident that the effective DAMGO concentration around the micropipette tip could be in a range representative (at least for the lower concentration) of that reached within the respiratory network after systemic administration, especially in case of opioid overdose. The result that 5-HT causes tachypneic effects on respiratory motor output through an action on preBötC neurons is in keeping with the vast majority of data obtained in neonatal and juvenile in situ rodent preparations. However, somewhat at variance with these previous studies, 5-HT-induced responses in the rabbit are mediated only by $5-\mathrm{HT}_{1 \mathrm{~A}}$ and $5-\mathrm{HT}_{3}$ receptors. Increases in breathing rate induced by the activation of $5-\mathrm{HT}_{1 \mathrm{~A}}$ receptors involve a disinhibition of preBötC GABAergic neurons. The presence of a disinhibitory mechanism is in general agreement with the results of previous rodent studies where an involvement of glycinergic neurons has been found. Since different animal species display similarities, but also differences, comparative studies on the control of breathing contribute to a better understanding of the respiratory CPG.

\section{Authors contributions}

All Authors prepared figures, drafted manuscript, edited and revised manuscript and approved final version of the manuscript.

\section{Acknowledgements}

This study was supported by grants from the University of Florence and from the Ente Cassa di Risparmio Firenze, Italy.

\section{References}

Abbott, S.B., Stornetta, R.L., Coates, M.B., Guyenet, P.G., 2011. Phox2b-expressing neurons of the parafacial region regulate breathing rate, inspiration, and expiration in conscious rats. J. Neurosci. 31, 16410-16422.

Abdala, A.P., Rybak, I.A., Smith, J.C., Paton, J.F., 2009. Abdominal expiratory activity in the rat brainstem-spinal cord in situ: patterns, origins and implications for respiratory rhythm generation. J. Physiol. 587, 3539-3559.

Al Zubaidy, Z.A., Erickson, R.L., Greer, J.J., 1996. Serotonergic and noradrenergic effects on respiratory neural discharge in the medullary slice preparation of neonatal rats. Pflügers Arch. 431, 942-949.

Alheid, G.F., McCrimmon, D.R., 2008. The chemical neuroanatomy of breathing. Respir. Physiol. Neurobiol. 164, 3-11.

Alheid, G.F., Gray, P.A., Jiang, M.C., Feldman, J.L., McCrimmon, D.R., 2002. Parvalbumin in respiratory neurons of the ventrolateral medulla of the adult rat. J. Neurocytol. 31, 693-717.

Alheid, G.F., Milsom, W.K., McCrimmon, D.R., 2004. Pontine influences on breathing: an overview. Respir. Physiol. Neurobiol. 143, 105-114.

Anderson, T.M., Ramirez, J.M., 2017. Respiratory rhythm generation: triple oscillator hypothesis. F1000Res. 6, 139.

Anderson, T.M., Garcia III, A.J., Baertsch, N.A., Pollak, J., Bloom, J.C., Wei, A.D., Rai, K. G., Ramirez, J.M., 2016. A novel excitatory network for the control of breathing. Nature 536, 76-80.

Ashhad, S., Feldman, J.L., 2020. Emergent elements of inspiratory rhythmogenesis: network synchronization and synchrony propagation. Neuron 106 (482-497), e484.

Bachmutsky, I., Wei, X.P., Kish, E., Yackle, K., 2020. Opioids depress breathing through two small brainstem sites. Elife 9.

Baertsch, N.A., Baertsch, H.C., Ramirez, J.M., 2018. The interdependence of excitation and inhibition for the control of dynamic breathing rhythms. Nat. Commun. 9, 843. 
Baertsch, N.A., Severs, L.J., Anderson, T.M., Ramirez, J.M., 2019. A spatially dynamic network underlies the generation of inspiratory behaviors. Proc. Natl. Acad. Sci. U.S. A. $116,7493-7502$.

Bianchi, A.L., Denavit-Saubié, M., Champagnat, J., 1995. Central control of breathing in mammals: neuronal circuitry, membrane properties, and neurotransmitters. Physiol Rev. 75, 1-45.

Bongianni, F., Mutolo, D., Pantaleo, T., 1997. Depressant effects on inspiratory and expiratory activity produced by chemical activation of Bötzinger complex neurons in the rabbit. Brain Res. 749, 1-9.

Bongianni, F., Mutolo, D., Carfi, M., Pantaleo, T., 2002. Respiratory responses to ionotropic glutamate receptor antagonists in the ventral respiratory group of the rabbit. Pflugers Arch. 444, 602-609.

Bongianni, F., Mutolo, D., Cinelli, E., Pantaleo, T., 2008. Neurokinin receptor modulation of respiratory activity in the rabbit. Eur. J. Neurosci. 27, 3233-3243.

Bongianni, F., Mutolo, D., Cinelli, E., Pantaleo, T., 2010. Respiratory responses induced by blockades of GABA and glycine receptors within the Bötzinger complex and the pre-Bötzinger complex of the rabbit. Brain Res. 1344, 134-147.

Burke, P.G., Abbott, S.B., McMullan, S., Goodchild, A.K., Pilowsky, P.M., 2010. Somatostatin selectively ablates post-inspiratory activity after injection into the Bötzinger complex. Neuroscience 167, 528-539.

Cinelli, E., Bongianni, F., Pantaleo, T., Mutolo, D., 2020. Activation of mu-opioid receptors differentially affects the preBötzinger Complex and neighbouring regions of the respiratory network in the adult rabbit. Respir. Physiol. Neurobiol. 280, 103482.

Connelly, C.A., Dobbins, E.G., Feldman, J.L., 1992. Pre-Bötzinger complex in cats: respiratory neuronal discharge patterns. Brain Res. 590, 337-340.

Cook-Snyder, D.R., Miller, J.R., Navarrete-Opazo, A.A., Callison, J.J., Peterson, R.C., Hopp, F.A., Stuth, E.A.E., Zuperku, E.J., Stucke, A.G., 2019. The contribution of endogenous glutamatergic input in the ventral respiratory column to respiratory rhythm. Respir. Physiol. Neurobiol. 260, 37-52.

Corcoran, A.E., Commons, K.G., Wu, Y., Smith, J.C., Harris, M.B., Richerson, G.B., 2014 Dual effects of 5-HT(1a) receptor activation on breathing in neonatal mice. J. Neurosci. 34, 51-59.

Cui, Y., Kam, K., Sherman, D., Janczewski, W.A., Zheng, Y., Feldman, J.L., 2016. Defining preBötzinger complex rhythm- and pattern-generating neural microcircuits in vivo. Neuron 91, 602-614.

Del Negro, C.A., Funk, G.D., Feldman, J.L., 2018. Breathing matters. Nat. Rev. Neurosci. $19,351-367$.

Depuy, S.D., Kanbar, R., Coates, M.B., Stornetta, R.L., Guyenet, P.G., 2011. Control of breathing by raphe obscurus serotonergic neurons in mice. J. Neurosci. 31, 1981-1990.

Dhingra, R.R., Dutschmann, M., Galan, R.F., Dick, T.E., 2017. Kolliker-Fuse nuclei regulate respiratory rhythm variability via a gain-control mechanism. Am. J. Physiol. Regul. Integr. Comp. Physiol. 312, R172-R188.

Dhingra, R.R., Furuya, W.I., Bautista, T.G., Dick, T.E., Galan, R.F., Dutschmann, M., 2019. Increasing local excitability of brainstem respiratory nuclei reveals a distributed network underlying respiratory motor pattern formation. Front. Physiol. 10,887 .

Dhingra, R.R., Dick, T.E., Furuya, W.I., Galan, R.F., Dutschmann, M., 2020. Volumetric mapping of the functional neuroanatomy of the respiratory network in the perfused brainstem preparation of rats. J. Physiol. 598, 2061-2079.

Doi, A., Ramirez, J.M., 2008. Neuromodulation and the orchestration of the respiratory rhythm. Respir. Physiol. Neurobiol. 164, 96-104.

Dutschmann, M., Dick, T.E., 2012. Pontine mechanisms of respiratory control. Compr. Physiol. 2, 2443-2469.

Dutschmann, M., Waki, H., Manzke, T., Simms, A.E., Pickering, A.E., Richter, D.W., Paton, J.F., 2009. The potency of different serotonergic agonists in counteracting opioid evoked cardiorespiratory disturbances. Philos. Trans. R. Soc. Lond., B, Biol. Sci. 364, 2611-2623.

Feldman, J.L., Del Negro, C.A., 2006. Looking for inspiration: new perspectives on respiratory rhythm. Nat. Rev. Neurosci. 7, 232-242.

Feldman, J.L., Connely, C.A., Ellenberger, H.H., Smith, J.C., 1990. The cardiorespiratory circuitry within the brainstem. Abstracts of the 13th Annual Meeting of the European Neuroscience Association 171.

Feldman, J.L., Mitchell, G.S., Nattie, E.E., 2003. Breathing: rhythmicity, plasticity, chemosensitivity. Annu. Rev. Neurosci. 26, 239-266.

Feldman, J.L., Del Negro, C.A., Gray, P.A., 2013. Understanding the rhythm of breathing: so near, yet so far. Annu. Rev. Physiol. 75, 423-452.

Fong, A.Y., Potts, J.T., 2006. Neurokinin-1 receptor activation in Bötzinger complex evokes bradypnoea. J. Physiol. 575, 869-885.

Funk, G.D., Smith, J.C., Feldman, J.L., 1993. Generation and transmission of respiratory oscillations in medullary slices: role of excitatory amino acids. J. Neurophysiol. 70, 1497-1515.

Furuya, W.I., Dhingra, R.R., Trevizan-Baú, P., McAllen, R.M., Dutschmann, M., 2021 The role of glycinergic inhibition in respiratory pattern formation and cardiorespiratory coupling in rats. Curr. Res, in Physiol. 4, 80-93.

Ge, Q., Feldman, J.L., 1998. AMPA receptor activation and phosphatase inhibition affect neonatal rat respiratory rhythm generation. J. Physiol. 509, 255-266.

Gray, P.A., Rekling, J.C., Bocchiaro, C.M., Feldman, J.L., 1999. Modulation of respiratory frequency by peptidergic input to rhythmogenic neurons in the preBötzinger complex. Science 286, 1566-1568.

Gray, P.A., Janczewski, W.A., Mellen, N., McCrimmon, D.R., Feldman, J.L., 2001. Normal breathing requires preBötzinger complex neurokinin-1 receptor- expressing neurons. Nat. Neurosci. 4, 927-930.
Gray, P.A., Hayes, J.A., Ling, G.Y., Llona, I., Tupal, S., Picardo, M.C., Ross, S.E., Hirata, T., Corbin, J.G., Eugenin, J., Del Negro, C.A., 2010. Developmental origin of preBötzinger complex respiratory neurons. J. Neurosci. 30, 14883-14895.

Greer, J.J., Smith, J.C., Feldman, J.L., 1991. Role of excitatory amino acids in the generation and transmission of respiratory drive in neonatal rat. J. Physiol. 437, 727-749.

Gromysz, H., Karczewski, W.A., 1981. Respiratory activity generated by a split brainstem preparation of the rabbit. Acta Neurobiol. Exp. (Wars.) 41, 237-242.

Gunther, S., Maroteaux, L., Schwarzacher, S.W., 2006. Endogenous 5-HT2B receptor activation regulates neonatal respiratory activity in vitro. J. Neurobiol. 66, 949-961.

Guyenet, P., 2012. How does CO2 activate the neurons of the retrotrapezoid nucleus? J. Physiol. 590, 2183-2184.

Guyenet, P.G., Stornetta, R.L., Abbott, S.B., Depuy, S.D., Kanbar, R., 2012. The retrotrapezoid nucleus and breathing. Adv. Exp. Med. Biol. 758, 115-122.

Haji, A., Takeda, R., Okazaki, M., 2000. Neuropharmacology of control of respiratory rhythm and pattern in mature mammals. Pharmacol. Ther. 86, 277-304.

Hayes, J.A., Del Negro, C.A., 2007. Neurokinin receptor-expressing pre-Bötzinger complex neurons in neonatal mice studied in vitro. J. Neurophysiol. 97, 4215-4224.

Hilaire, G., Duron, B., 1999. Maturation of the mammalian respiratory system. Physiol. Rev. 79, 325-360.

Hilaire, G., Voituron, N., Menuet, C., Ichiyama, R.M., Subramanian, H.H., Dutschmann, M., 2010. The role of serotonin in respiratory function and dysfunction. Respir. Physiol. Neurobiol. 174, 76-88.

Hodges, M.R., Richerson, G.B., 2008. Contributions of 5-HT neurons to respiratory control: neuromodulatory and trophic effects. Respir. Physiol. Neurobiol. 164, $222-232$.

Hodges, M.R., Richerson, G.B., 2010. The role of medullary serotonin (5-HT) neurons in respiratory control: contributions to eupneic ventilation, CO2 chemoreception, and thermoregulation. J. Appl. Physiol. 108, 1425-1432.

Hülsmann, S., Hagos, L., Eulenburg, V., Hirrlinger, J., 2021. Inspiratory off-switch mediated by optogenetic activation of inhibitory neurons in the preBötzinger complex in vivo. Int. J. Mol. Sci. 22.

Iovino, L., Mutolo, D., Cinelli, E., Contini, M., Pantaleo, T., Bongianni, F., 2019. Breathing stimulation mediated by 5-HT1A and 5-HT3 receptors within the preBötzinger complex of the adult rabbit. Brain Res. 1704, 26-39.

Janczewski, W.A., Feldman, J.L., 2006. Distinct rhythm generators for inspiration and expiration in the juvenile rat. J. Physiol. 570, 407-420.

Janczewski, W.A., Tashima, A., Hsu, P., Cui, Y., Feldman, J.L., 2013. Role of inhibition in respiratory pattern generation. J. Neurosci. 33, 5454-5465.

Jiang, C., Shen, E., 1991. Respiratory neurons in the medulla of the rabbit: distribution, discharge patterns and spinal projections. Brain Res. 541, 284-292.

Johnson, S.M., Smith, J.C., Funk, G.D., Feldman, J.L., 1994. Pacemaker behavior of respiratory neurons in medullary slices from neonatal rat. J. Neurophysiol. 72 , 2598-2608.

Johnson, S.M., Koshiya, N., Smith, J.C., 2001. Isolation of the kernel for respiratory rhythm generation in a novel preparation: the pre-Bötzinger complex "island". J. Neurophysiol. 85, 1772-1776.

Jones, S.E., Dutschmann, M., 2016. Testing the hypothesis of neurodegeneracy in respiratory network function with a priori transected arterially perfused brain stem preparation of rat. J. Neurophysiol. 115, 2593-2607.

Kam, K., Worrell, J.W., Janczewski, W.A., Cui, Y., Feldman, J.L., 2013. Distinct inspiratory rhythm and pattern generating mechanisms in the preBötzinger complex. J. Neurosci. 33, 9235-9245.

Krause, K.L., Neumueller, S.E., Marshall, B.D., Kiner, T., Bonis, J.M., Pan, L.G., Qian, B., Forster, H.V., 2009. $\mu$-opioid receptor agonist injections into the presumed preBötzinger complex and the surrounding region of awake goats do not alter eupneic breathing. J. Appl. Physiol. 107, 1591-1599.

Leresche, N., Asprodini, E., Emri, Z., Cope, D.W., Crunelli, V., 2000. Somatostatin inhibits GABAergic transmission in the sensory thalamus via presynaptic receptors. Neuroscience 98, 513-522.

Levitt, E.S., Abdala, A.P., Paton, J.F., Bissonnette, J.M., Williams, J.T., 2015. Mu opioid receptor activation hyperpolarizes respiratory-controlling Kölliker-Fuse neurons and suppresses post-inspiratory drive. J. Physiol. 593, 4453-4469.

Lipski, J., Bellingham, M.C., West, M.J., Pilowsky, P., 1988. Limitations of the technique of pressure microinjection of excitatory amino acids for evoking responses from localized regions of the CNS. J. Neurosci. Methods 26, 169-179.

Llona, I., Eugenin, J., 2005. Central actions of somatostatin in the generation and control of breathing. Biol. Res. 38, 347-352.

Llona, I., Ampuero, E., Eugenin, J.L., 2004. Somatostatin inhibition of fictive respiration is modulated by pH. Brain Res. 1026, 136-142.

Lonergan, T., Goodchild, A.K., Christie, M.J., Pilowsky, P.M., 2003. Mu opioid receptors in rat ventral medulla: effects of endomorphin-1 on phrenic nerve activity. Respir. Physiol. Neurobiol. 138, 165-178.

Manzke, T., Dutschmann, M., Schlaf, G., Morschel, M., Koch, U.R., Ponimaskin, E., Bidon, O., Lalley, P.M., Richter, D.W., 2009. Serotonin targets inhibitory synapses to induce modulation of network functions. Philos. Trans. R. Soc. Lond. B Biol. Sci. 364, 2589-2602.

McCrimmon, D.R., Monnier, A., Hayashi, F., Zuperku, E.J., 2000. Pattern formation and rhythm generation in the ventral respiratory group. Clin. Exp. Pharmacol. Physiol. 27, 126-131.

McKay, L.C., Janczewski, W.A., Feldman, J.L., 2005. Sleep-disordered breathing after targeted ablation of preBötzinger complex neurons. Nat. Neurosci. 8, 1142-1144.

Mellen, N.M., Janczewski, W.A., Bocchiaro, C.M., Feldman, J.L., 2003. Opioid-induced quantal slowing reveals dual networks for respiratory rhythm generation. Neuron $37,821-826$. 
Menuet, C., Connelly, A.A., Bassi, J.K., Melo, M.R., Le, S., Kamar, J., Kumar, N.N., McDougall, S.J., McMullan, S., Allen, A.M., 2020. PreBötzinger complex neurons drive respiratory modulation of blood pressure and heart rate. Elife 9 .

Michelsen, L.G., Salmenpera, M., Hug Jr., C.C., Szlam, F., VanderMeer, D., 1996. Anesthetic potency of remifentanil in dogs. Anesthesiology 84, 865-872.

Miller, J.R., Zuperku, E.J., Stuth, E.A.E., Banerjee, A., Hopp, F.A., Stucke, A.G., 2017. A subregion of the parabrachial nucleus partially mediates respiratory rate depression from intravenous remifentanil in young and adult rabbits. Anesthesiology 127, 502-514.

Montandon, G., Qin, W., Liu, H., Ren, J., Greer, J.J., Horner, R.L., 2011. PreBötzinger complex neurokinin-1 receptor-expressing neurons mediate opioid-induced respiratory depression. J. Neurosci. 31, 1292-1301.

Montandon, G., Liu, H., Horner, R.L., 2016a. Contribution of the respiratory network to rhythm and motor output revealed by modulation of GIRK channels, somatostatin and neurokinin-1 receptors. Sci. Rep. 6, 32707.

Montandon, G., Ren, J., Victoria, N.C., Liu, H., Wickman, K., Greer, J.J., Horner, R.L., 2016b. G-protein-gated inwardly rectifying potassium channels modulate respiratory depression by opioids. Anesthesiology 124, 641-650.

Morgado-Valle, C., Feldman, J.L., 2007. NMDA receptors in preBötzinger complex neurons can drive respiratory rhythm independent of AMPA receptors. J. Physiol. 582, 359-368.

Mulkey, D.K., Stornetta, R.L., Weston, M.C., Simmons, J.R., Parker, A., Bayliss, D.A., Guyenet, P.G., 2004. Respiratory control by ventral surface chemoreceptor neurons in rats. Nat. Neurosci. 7, 1360-1369.

Mustapic, S., Radocaj, T., Sanchez, A., Dogas, Z., Stucke, A.G., Hopp, F.A., Stuth, E.A., Zuperku, E.J., 2010. Clinically relevant infusion rates of mu-opioid agonist remifentanil cause bradypnea in decerebrate dogs but not via direct effects in the pre-Bötzinger complex region. J. Neurophysiol. 103, 409-418.

Mutolo, D., Bongianni, F., Carfi, M., Pantaleo, T., 2002. Respiratory changes induced by kainic acid lesions in rostral ventral respiratory group of rabbits. Am. J. Physiol Regul. Integr. Comp. Physiol. 283, R227-R242.

Mutolo, D., Bongianni, F., Nardone, F., Pantaleo, T., 2005. Respiratory responses evoked by blockades of ionotropic glutamate receptors within the Bötzinger complex and the pre-Bötzinger complex of the rabbit. Eur. J. Neurosci. 21, 122-134.

Nichols, D.E., Nichols, C.D., 2008. Serotonin receptors. Chem. Rev. 108, 1614-1641.

Nicholson, C., Sykova, E., 1998. Extracellular space structure revealed by diffusion analysis. Trends Neurosci. 21, 207-215.

Niebert, M., Vogelgesang, S., Koch, U.R., Bischoff, A.M., Kron, M., Bock, N., Manzke, T., 2011. Expression and function of serotonin $2 \mathrm{~A}$ and $2 \mathrm{~B}$ receptors in the mammalian respiratory network. PLoS One 6, e21395.

Onimaru, H., Homma, I., 1992. Whole cell recordings from respiratory neurons in the medulla of brainstem-spinal cord preparations isolated from newborn rats. Pflügers Arch. 420, 399-406.

Onimaru, H., Homma, I., 2003. A novel functional neuron group for respiratory rhythm generation in the ventral medulla. J. Neurosci. 23, 1478-1486.

Pagliardini, S., Janczewski, W.A., Tan, W.B., Dickson, C.T., Deisseroth, K., Feldman, J.L. 2011. Active expiration induced by excitation of ventral medulla in adult anesthetized rats. J. Neurosci. 31, 2895-2905.

Pantaleo, T., Mutolo, D., Cinelli, E., Bongianni, F., 2011. Respiratory responses to somatostatin microinjections into the Bötzinger complex and the pre-Bötzinger complex of the rabbit. Neurosci. Lett. 498, 26-30.

Paterson, D.S., Hilaire, G., Weese-Mayer, D.E., 2009. Medullary serotonin defects and respiratory dysfunction in sudden infant death syndrome. Respir. Physiol. Neurobiol. 168, 133-143.

Peña, F., Ramirez, J.M., 2002. Endogenous activation of serotonin-2A receptors is required for respiratory rhythm generation in vitro. J. Neurosci. 22, 11055-11064.

Peña, F., Ramirez, J.M., 2004. Substance P-mediated modulation of pacemaker properties in the mammalian respiratory network. J. Neurosci. 24, 7549-7556.

Pennefather, J.N., Lecci, A., Candenas, M.L., Patak, E., Pinto, F.M., Maggi, C.A., 2004. Tachykinins and tachykinin receptors: a growing family. Life Sci. 74, 1445-1463.

Pierrefiche, O., Schwarzacher, S.W., Bischoff, A.M., Richter, D.W., 1998. Blockade of synaptic inhibition within the pre-Bötzinger complex in the cat suppresses respiratory rhythm generation in vivo. J. Physiol. 509, 245-254.

Prkic, I., Mustapic, S., Radocaj, T., Stucke, A.G., Stuth, E.A., Hopp, F.A., Dean, C., Zuperku, E.J., 2012. Pontine mu-opioid receptors mediate bradypnea caused by intravenous remifentanil infusions at clinically relevant concentrations in dogs. J. Neurophysiol. 108, 2430-2441.

Ptak, K., Yamanishi, T., Aungst, J., Milescu, L.S., Zhang, R., Richerson, G.B., Smith, J.C. 2009. Raphe neurons stimulate respiratory circuit activity by multiple mechanisms via endogenously released serotonin and substance P. J. Neurosci. 29, 3720-3737.

Qi, J., Li, H., Zhao, T.B., Lu, Y.C., Zhang, T., Li, J.L., Dong, Y.L., Li, Y.Q., 2017. Inhibitory effect of Endomorphin-2 binding to the mu-opioid receptor in the rat pre-bötzinger complex on the breathing activity. Mol. Neurobiol. 54, 461-469.

Ramirez, J.M., Baertsch, N.A., 2018. The dynamic basis of respiratory rhythm generation: one breath at a time. Annu. Rev. Neurosci. 41, 475-499.

Ramirez, J.M., Schwarzacher, S.W., Pierrefiche, O., Olivera, B.M., Richter, D.W., 1998. Selective lesioning of the cat pre-Bötzinger complex in vivo eliminates breathing but not gasping. J. Physiol. 507, 895-907.

Ramirez, J.M., Doi, A., Garcia 3rd, A.J., Elsen, F.P., Koch, H., Wei, A.D., 2012. The cellular building blocks of breathing. Compr. Physiol. 2, 2683-2731.

Ramirez, J.M., Garcia III, A.J., Anderson, T.M., Koschnitzky, J.E., Peng, Y.J., Kumar, G. K., Prabhakar, N.R., 2013. Central and peripheral factors contributing to obstructive sleep apneas. Respir. Physiol. Neurobiol. 189, 344-353.

Ramirez, J.M., Dashevskiy, T., Marlin, I.A., Baertsch, N., 2016. Microcircuits in respiratory rhythm generation: commonalities with other rhythm generating networks and evolutionary perspectives. Curr. Opin. Neurobiol. 41, 53-61.
Ramirez-Jarquin, J.O., Lara-Hernandez, S., Lopez-Guerrero, J.J., Aguileta, M.A., RiveraAngulo, A.J., Sampieri, A., Vaca, L., Ordaz, B., Pena-Ortega, F., 2012. Somatostatin modulates generation of inspiratory rhythms and determines asphyxia survival. Peptides 34, 360-372.

Rekling, J.C., Feldman, J.L., 1998. PreBötzinger complex and pacemaker neurons: hypothesized site and kernel for respiratory rhythm generation. Annu. Rev. Physiol. 60, 385-405.

Richter, D.W., Ballantyne, D., Remmers, J.E., 1986. How is the respiratory rhythm generated? A model. News Physiol. Sci. 1, 109-112.

Rybak, I.A., Abdala, A.P., Markin, S.N., Paton, J.F., Smith, J.C., 2007. Spatial organization and state-dependent mechanisms for respiratory rhythm and pattern generation. Prog. Brain Res. 165, 201-220.

Rybak, I.A., O'Connor, R., Ross, A., Shevtsova, N.A., Nuding, S.C., Segers, L.S., Shannon, R., Dick, T.E., Dunin-Barkowski, W.L., Orem, J.M., Solomon, I.C., Morris, K.F., Lindsey, B.G., 2008. Reconfiguration of the pontomedullary respiratory network: a computational modeling study with coordinated in vivo experiments. J. Neurophysiol. 100, 1770-1799.

Schwarzacher, S.W., Smith, J.C., Richter, D.W., 1995. Pre-Bötzinger complex in the cat. J. Neurophysiol. 73, 1452-1461.

Schwarzacher, S.W., Pestean, A., Gunther, S., Ballanyi, K., 2002. Serotonergic modulation of respiratory motoneurons and interneurons in brainstem slices of perinatal rats. Neuroscience 115, 1247-1259.

Schwarzacher, S.W., Rub, U., Deller, T., 2011. Neuroanatomical characteristics of the human pre-Bötzinger complex and its involvement in neurodegenerative brainstem diseases. Brain 134, 24-35.

Severini, C., Improta, G., Falconieri-Erspamer, G., Salvadori, S., Erspamer, V., 2002. The tachykinin peptide family. Pharmacol. Rev. 54, 285-322.

Shao, J., Tsao, T.H., Butera, R., 2006. Bursting without slow kinetics: a role for a small world? Neural Comput. 18, 2029-2035.

Shek, J.W., Wen, G.Y., Wisniewski, H.M., 1986. Atlas of the Rabbit Brain and Spinal Cord. Karger, Basel.

Sherman, D., Worrell, J.W., Cui, Y., Feldman, J.L., 2015. Optogenetic perturbation of preBötzinger complex inhibitory neurons modulates respiratory pattern. Nat. Neurosci. 18, 408-414.

Shevtsova, N.A., Manzke, T., Molkov, Y.I., Bischoff, A., Smith, J.C., Rybak, I.A., Richter, D.W., 2011. Computational modelling of 5-HT receptor-mediated reorganization of the brainstem respiratory network. Eur. J. Neurosci. 34, 1276-1291.

Smith, J.C., Ellenberger, H.H., Ballanyi, K., Richter, D.W., Feldman, J.L., 1991. PreBötzinger complex: a brainstem region that may generate respiratory rhythm in mammals. Science 254, 726-729.

Smith, J.C., Abdala, A.P., Koizumi, H., Rybak, I.A., Paton, J.F., 2007. Spatial and functional architecture of the mammalian brain stem respiratory network: a hierarchy of three oscillatory mechanisms. J. Neurophysiol. 98, 3370-3387.

Smith, J.C., Abdala, A.P., Rybak, I.A., Paton, J.F., 2009. Structural and functional architecture of respiratory networks in the mammalian brainstem. Philos. Trans. R. Soc. Lond B Biol. Sci. 364, 2577-2587.

Smith, J.C., Abdala, A.P., Borgmann, A., Rybak, I.A., Paton, J.F., 2013. Brainstem respiratory networks: building blocks and microcircuits. Trends Neurosci. 36, $152-162$.

Solomon, I.C., 2000. Excitation of phrenic and sympathetic output during acute hypoxia: contribution of medullary oxygen detectors. Respir. Physiol. 121, 101-117.

Spary, E.J., Maqbool, A., Batten, T.F., 2008. Expression and localisation of somatostatin receptor subtypes sst1-sst5 in areas of the rat medulla oblongata involved in autonomic regulation. J. Chem. Neuroanat. 35, 49-66.

St Jacques, R., St John, W.M., 1999. Transient, reversible apnoea following ablation of the pre-Bötzinger complex in rats. J. Physiol. 520, 303-314.

Stucke, A.G., Miller, J.R., Prkic, I., Zuperku, E.J., Hopp, F.A., Stuth, E.A., 2015. Opioid induced respiratory depression is only partially mediated by the preBötzinger complex in young and adult rabbits in vivo. Anesthesiology 122, 1288-1298.

Sun, Q.J., Goodchild, A.K., Chalmers, J.P., Pilowsky, P.M., 1998. The pre-Bötzinger complex and phase-spanning neurons in the adult rat. Brain Res. 809, 204-213.

Sun, X., Thorn Perez, C., Halemani, D.N., Shao, X.M., Greenwood, M., Heath, S., Feldman, J.L., Kam, K., 2019. Opioids modulate an emergent rhythmogenic process to depress breathing. Elife 8.

Tan, W., Janczewski, W.A., Yang, P., Shao, X.M., Callaway, E.M., Feldman, J.L., 2008. Silencing preBötzinger complex somatostatin-expressing neurons induces persistent apnea in awake rat. Nat. Neurosci. 11, 538-540.

Tan, W., Pagliardini, S., Yang, P., Janczewski, W.A., Feldman, J.L., 2010. Projections of preBötzinger Complex neurons in adult rats. J. Comp. Neurol. 518, 1862-1878.

Teran, F.A., Massey, C.A., Richerson, G.B., 2014. Chapter 11 - Serotonin neurons and central respiratory chemoreception: where are we now? In: Holstege, G., Beers, C.M., Subramanian, H.H. (Eds.), Progress in Brain Research. Elsevier, pp. 207-233.

Toor, R., Sun, Q.J., Kumar, N.N., Le, S., Hildreth, C.M., Phillips, J.K., McMullan, S., 2019. Neurons in the intermediate reticular nucleus coordinate postinspiratory activity, swallowing, and respiratory-sympathetic coupling in the rat. J. Neurosci. 39, 9757-9766.

Varga, A.G., Reid, B.T., Kieffer, B.L., Levitt, E.S., 2020. Differential impact of two critical respiratory centres in opioid-induced respiratory depression in awake mice. J. Physiol. 598, 189-205.

Von Euler, C., 1986. Neurophysiology of breathing in mammals. In: Bloom, F.E. (Ed.), Handbook of Physiology. The Nervous System IV. American Physiological Society, Bethesda, pp. 463-524.

Von Euler, C., 1997. Neural organization and rhythm generation. In: Crystal, R.G., West, J.B., Barnes, P.J., Weibel, E.R. (Eds.), The Lung: Scientific Foundations, second edition. Lippincott-Raven, Philadelphia, pp. 1711-1724. 
Wallen-Mackenzie, A., Gezelius, H., Thoby-Brisson, M., Nygard, A., Enjin, A., Fujiyama, F., Fortin, G., Kullander, K., 2006. Vesicular glutamate transporter 2 is required for central respiratory rhythm generation but not for locomotor central pattern generation. J. Neurosci. 26, 12294-12307.

Wallen-Mackenzie, A., Wootz, H., Englund, H., 2010. Genetic inactivation of the vesicular glutamate transporter 2 (VGLUT2) in the mouse: what have we learnt about functional glutamatergic neurotransmission? Ups. J. Med. Sci. 115, 11-20.

Wang, H., Germanson, T.P., Guyenet, P.G., 2002. Depressor and tachypneic responses to chemical stimulation of the ventral respiratory group are reduced by ablation of neurokinin-1 receptor-expressing neurons. J. Neurosci. 22, 3755-3764.

Wang, S., Shi, Y., Shu, S., Guyenet, P.G., Bayliss, D.A., 2013. Phox2b-expressing retrotrapezoid neurons are intrinsically responsive to $\mathrm{H}+$ and $\mathrm{CO} 2$. J. Neurosci. 33, $7756-7761$.
Wenninger, J.M., Pan, L.G., Klum, L., Leekley, T., Bastastic, J., Hodges, M.R., Feroah, T., Davis, S., Forster, H.V., 2004a. Small reduction of neurokinin-1 receptor-expressing neurons in the pre-Bötzinger complex area induces abnormal breathing periods in awake goats. J. Appl. Physiol. 97, 1620-1628.

Wenninger, J.M. Pan, L.G., Klum, L., Leekley, T., Bastastic, J., Hodges, M.R., Feroah, T. R., Davis, S., Forster, H.V., 2004b. Large lesions in the pre-Bötzinger complex area eliminate eupneic respiratory rhythm in awake goats. J. Appl. Physiol. 97, 1629-1636.

Yamamoto, Y., Lagercrantz, H., 1985. Some effects of substance P on central respiratory control in rabbit pups. Acta Physiol. Scand. 124, 449-455.

Yang, C.F., Feldman, J.L., 2018. Efferent projections of excitatory and inhibitory preBötzinger Complex neurons. J. Comp. Neurol. 526, 1389-1402.

Yang, C.F., Kim, E.J., Callaway, E.M., Feldman, J.L., 2020. Monosynaptic projections to excitatory and inhibitory preBötzinger complex neurons. Front. Neuroanat. 14, 58. 